\title{
Poisson-Lie plurals of Bianchi cosmologies and Generalized Supergravity Equations
}

\author{
Ladislav Hlavatý $\dot{y}^{a}$ and Ivo Petr ${ }^{b}$ \\ ${ }^{a}$ Department of Physics, Faculty of Nuclear Sciences and Physical Engineering, \\ Czech Technical University in Prague, \\ Břehová 7, Prague 1, 115 19, Czech Republic \\ ${ }^{b}$ Department of Applied Mathematics, Faculty of Information Technology, \\ Czech Technical University in Prague, \\ Thákurova 9, Prague 6, 160 00, Czech Republic \\ E-mail: hlavaty@fjfi.cvut.cz, ivo.petr@fit.cvut.cz
}

ABStract: Poisson-Lie T-duality and plurality are important solution generating techniques in string theory and (generalized) supergravity. Since duality/plurality does not preserve conformal invariance, the usual beta function equations are replaced by Generalized Supergravity Equations containing vector $\mathcal{J}$. In this paper we apply Poisson-Lie T-plurality on Bianchi cosmologies. We present a formula for the vector $\mathcal{J}$ as well as transformation rule for dilaton, and show that plural backgrounds together with this dilaton and $\mathcal{J}$ satisfy the Generalized Supergravity Equations. The procedure is valid also for non-local dilaton and non-constant $\mathcal{J}$. We also show that $\operatorname{Div} \Theta$ of the non-commutative structure $\Theta$ used for non-Abelian T-duality or integrable deformations does not give correct $\mathcal{J}$ for Poisson-Lie T-plurality.

KEYwords: Sigma Models, String Duality, Supergravity Models, Integrable Field Theories

ArXiv EPrint: 1910.08436 


\section{Contents}

1 Introduction 1

2 Basics of Poisson-Lie T-plurality 3

2.1 Sigma models 3

2.2 Formulas for Poisson-Lie T-plurality with spectators 4

2.3 Generalized Supergravity Equations and transformation of dilaton 6

3 Poisson-Lie T-plurality of flat background $\quad 7$

3.1 Bianchi $I I I$ cosmology $\quad 7$

3.1.1 Transformation of $\mathfrak{b}_{I I I} \bowtie \mathfrak{a}$ to $\mathfrak{b}_{I I I} \bowtie \mathfrak{b}_{I I}$ and to its dual

3.1.2 Transformation of $\mathfrak{b}_{I I I} \bowtie \mathfrak{a}$ to $\mathfrak{b}_{I I I} \bowtie \mathfrak{b}_{\text {IIIiii }}$ and to its dual

$\begin{array}{lll}3.2 & \text { Bianchi } V \text { cosmology } & 13\end{array}$

3.2.1 Transformation of $\mathfrak{b}_{V} \bowtie \mathfrak{a}$ to $\mathfrak{b}_{V I_{-1}} \bowtie \mathfrak{a}$ and to its dual

$\begin{array}{lll}\text { 3.2.2 Transformation of } \mathfrak{b}_{V} \bowtie \mathfrak{a} \text { to } \mathfrak{b}_{V} \bowtie \mathfrak{b}_{I I} \text { and to its dual } & 15\end{array}$

3.2.3 Transformation of $\mathfrak{b}_{V} \bowtie \mathfrak{a}$ to $\mathfrak{b}_{V I_{-1}} \bowtie \mathfrak{b}_{V i i}$ and to its dual $\quad 18$

4 Poisson-Lie T-plurality of curved cosmologies 20

$\begin{array}{lll}4.1 & \text { Bianchi } V I_{-1} \text { cosmology } & 20\end{array}$

4.1.1 Transformation of $\mathfrak{b}_{V I_{-1}} \bowtie \mathfrak{a}$ to $\mathfrak{b}_{V} \bowtie \mathfrak{a}$ and to its dual 21

4.2 Bianchi $V I_{\kappa}$ cosmology 22

4.2.1 Transformation of $\mathfrak{b}_{V I_{\kappa}} \bowtie \mathfrak{a}$ to $\mathfrak{b}_{V I_{\kappa}} \bowtie \mathfrak{b}_{I I}$ and to its dual 23

4.2.2 Transformation of $\mathfrak{b}_{V I_{\kappa}} \bowtie \mathfrak{a}$ to $\mathfrak{b}_{V I_{\kappa}} \bowtie \mathfrak{b}_{V I_{-\kappa} . i i i}$ and to its dual $\quad 24$

5 Conclusions $\quad 26$

\section{Introduction}

In recent years we have seen renewed interest in (non-)Abelian T-duality [1, 2] of sigma models. Its generalization [3, 4] includes RR fields and is often used to find new supergravity solutions $[5,6]$. Duality transformation can be performed whenever there is an isometry of the sigma model background. However, dualization with respect to non-semisimple Lie groups $\mathscr{G}$ does not preserve conformal invariance [7] and dual models exhibit mixed gauge and gravitational anomaly proportional to the trace of structure constants of Lie algebra $\mathfrak{g}$ corresponding to $\mathscr{G}[8]$.

Non-Abelian T-duality also contributes to the study of integrable deformations $[9,10]$ where similar problem has been dealt with [11]. After publication of [12] it became clear that integrable deformations should satisfy the so-called Generalized Supergavity Equations 
instead of usual supergravity equations. Since some deformations can be understood as nonAbelian T-duals [13], one may ask whether dual models satisfy Generalized Supergravity Equations in general.

Authors of [14] have found non-Abelian T-duals of Bianchi cosmologies [15] and have shown that dual backgrounds indeed satisfy Generalised Supergravity Equations. In our recent paper [16] we noted that beside "full" non-Abelian T-dualities used in [14] there are other transformations that belong to the NATD-group investigated in [17]. Namely, we considered B-shifts, $\beta$-shifts, "factorized" dualities and their compositions and we have shown that backgrounds obtained by these transformations satisfy Generalized Supergravity Equations as well. The above mentioned transformations are best understood in context of Poisson-Lie T-duality [18] and Poisson-Lie T-plurality [19]. Underlying algebraic structure of these transformations is the Drinfel'd double $\mathscr{D}$ formed by a pair of Lie subgroups $\mathscr{G}$ and $\widetilde{\mathscr{G}}$. Corresponding Lie algebras form Manin triple $(\mathfrak{d}, \mathfrak{g}, \tilde{\mathfrak{g}})$.

Formulas summarized in section 2 allow us to construct sigma models on Lie groups $\mathscr{G}$ and $\widetilde{\mathscr{G}}$ that possess generalized symmetries [20] and satisfy Generalised Supergravity Equations for certain dilaton field. However, beside $\mathscr{G}$ and $\widetilde{\mathscr{G}}$ there might also exist other pairs of groups $\widehat{\mathscr{G}}$ and $\overline{\mathscr{G}}$ that form the same Drinfel'd double $\mathscr{D}$. It is then natural to ask what backgrounds on $\widehat{\mathscr{G}}$ or $\overline{\mathscr{G}}$ we get by Poisson-Lie T-plurality and whether these backgrounds satisfy the Generalised Supergravity Equations. For $A d S_{5} \times S^{5}$ background this problem was addressed in [21].

In this paper we find Poisson-Lie T-plurals of cosmologies invariant with respect to nonsimple Bianchi groups. To do so, we consider semi-Abelian Drinfel'd doubles $\mathscr{D}=(\mathscr{B} \mid \mathscr{A})$, where $\mathscr{B}$ are three-dimensional groups of symmetries of the particular cosmology and $\mathscr{A}$ is three-dimensional Abelian group. Manin triples of such Drinfel'd doubles were classified in [22]. It turns out that for each group $\mathscr{B}$ there are several Manin triples and many embedings of these Manin triples into $\mathfrak{d}$. Resulting plural backgrounds depend on many constants [16] and it is a tremendous task to put down complete classification. Since it is not always possible to present full results, we often restrict to particular examples of Poisson-Lie T-plurality to show important features of obtained models.

The main goal of the paper is to show that new backgrounds obtained by PoissonLie T-plurality satisfy Generalized Supergravity Equations. Straightforward application of dilaton transformation formula [19] gives dilatons on $\widehat{\mathscr{G}}$ and $\overline{\mathscr{G}}$ that may depend on coordinates of the dual group leading to puzzle discussed in [23]. Inspired by [21] we show how to deal with this issue and present formulas that allow us to calculate vector field $\mathcal{J}$ appearing in Generalized Supergravity Equations. The plural backgrounds, dilatons and $\mathcal{J}$ then satisfy these equations.

Authors of [24-26] consider Yang-Baxter deformations of $A d S_{5} \times S^{5}$ and calculate vector field $\mathcal{J}$ as $\operatorname{Div} \Theta$ from non-commutative structure $\Theta$ in the open string picture. This works for "full" non-Abelian dualities, but not for "factorized" dualities (e.g. some backgrounds obtained in [16]) or more general pluralities.

Plan of the paper is following. In next section we summarize basics of Poisson-Lie Tplurality including transformation of dilaton and write down formulas for vector field $\mathcal{J}$. In sections 3.1, 3.2 we derive metrics, B-fields and dilatons obtained by Poisson-Lie T-plurality 
of the flat background, and in sections 4.1, 4.2 we present metrics, B-fields and dilatons obtained by Poisson-Lie T-plurality of curved Bianchi cosmologies with nontrivial dilaton. It turns out that for appropriately defined vector field $\mathcal{J}$ all of them satisfy Generalized Supergravity Equations.

\section{Basics of Poisson-Lie T-plurality}

In the first two subsections we recapitulate Poisson-Lie T-plurality with spectators $[18,19$, 27].

\subsection{Sigma models}

Let $\mathscr{M}$ be $(n+d)$-dimensional (pseudo-)Riemannian target manifold and consider sigma model on $\mathscr{M}$ given by Lagrangian

$$
\mathcal{L}=\partial_{-} \phi^{\mu} \mathcal{F}_{\mu \nu}(\phi) \partial_{+} \phi^{\nu}, \quad \phi^{\mu}=\phi^{\mu}\left(\sigma_{+}, \sigma_{-}\right), \quad \mu=1, \ldots, n+d
$$

where tensor field $\mathcal{F}=\mathcal{G}+\mathcal{B}$ on $\mathscr{M}$ defines metric and torsion potential (Kalb-Ramond field) of the target manifold. Assume that there is a $d$-dimensional Lie group $\mathscr{G}$ with free action on $\mathscr{M}$ that leaves the tensor invariant. The action of $\mathscr{G}$ is transitive on its orbits, hence we may locally consider $\mathscr{M} \approx(\mathscr{M} / \mathscr{G}) \times \mathscr{G}=\mathscr{N} \times \mathscr{G}$, and introduce adapted coordinates $^{1}$

$$
\left\{s_{\alpha}, x^{a}\right\}, \quad \alpha=1, \ldots, n=\operatorname{dim} \mathscr{N}, \quad a=1, \ldots, d=\operatorname{dim} \mathscr{G}
$$

where $s_{\alpha}$ label the orbits of $\mathscr{G}$ and are treated as spectators, and $x^{a}$ are group coordinates. Dualizable sigma model on $\mathscr{N} \times \mathscr{G}$ is given by tensor field $\mathcal{F}$ defined by spectator-dependent $(n+d) \times(n+d)$ matrix $E(s)$ and group-dependent $\mathcal{E}(x)$ as

$$
\mathcal{F}(s, x)=\mathcal{E}(x) \cdot E(s) \cdot \mathcal{E}^{T}(x), \quad \mathcal{E}(x)=\left(\begin{array}{cc}
\mathbf{1}_{n} & 0 \\
0 & e(x)
\end{array}\right)
$$

where $e(x)$ is $d \times d$ matrix of components of right-invariant Maurer-Cartan form $(d g) g^{-1}$ on $\mathscr{G}$.

Using non-Abelian T-duality one can find dual sigma model on $\mathscr{N} \times \mathscr{A}$, where $\mathscr{A}$ is Abelian subgroup of semi-Abelian Drinfel'd double $\mathscr{D}=(\mathscr{G} \mid \mathscr{A})$. The necessary formulas will be given in the following subsection as a special case of Poisson-Lie T-plurality.

In this paper the groups $\mathscr{G}$ will be non-semisimple Bianchi groups. Their elements will be parametrized as $g=e^{x^{1} T_{1}} e^{x^{2} T_{2}} e^{x^{3} T_{3}}$ where $e^{x^{2} T_{2}} e^{x^{3} T_{3}}$ and $e^{x^{3} T_{3}}$ parametrize their normal subgroups. Bianchi cosmologies are defined on four-dimensional manifolds, hence $\operatorname{dim} \mathscr{N}=1$ and we denote the spectator $s_{1}$ as $t$.

\footnotetext{
${ }^{1}$ Detailed discussion of the process of finding adapted coordinates can be found e.g. in [27, 28].
} 


\subsection{Formulas for Poisson-Lie T-plurality with spectators}

Drinfel'd double $\mathscr{D}=(\mathscr{G} \mid \widetilde{\mathscr{G}})$ is a $2 d$-dimensional Lie group whose Lie algebra $\mathfrak{d}$ is equipped with an ad-invariant non-degenerate symmetric bilinear form $\langle.,$.$\rangle . The Lie algebra \mathfrak{d}$ decomposes into double cross sum $\mathfrak{g} \bowtie \tilde{\mathfrak{g}}$ of subalgebras $\mathfrak{g}$ and $\tilde{\mathfrak{g}}[29]$ that are maximally isotropic with respect to $\langle.,$.$\rangle giving rise to Manin triple (\mathfrak{d}, \mathfrak{g}, \tilde{\mathfrak{g}})$. This means that mutually dual bases $T_{a} \in \mathfrak{g}, \widetilde{T}^{a} \in \tilde{\mathfrak{g}}, a=1, \ldots, d$, satisfy relations

$$
\left\langle T_{a}, T_{b}\right\rangle=0, \quad\left\langle\widetilde{T}^{a}, \widetilde{T}^{b}\right\rangle=0, \quad\left\langle T_{a}, \widetilde{T}^{b}\right\rangle=\delta_{a}^{b},
$$

and due to ad-invariance of the bilinear form $\langle.,$.$\rangle the algebraic structure of Manin triple$ is given by

$$
\left[T_{i}, T_{j}\right]=f_{i j}^{k} T_{k}, \quad\left[\widetilde{T}^{i}, \widetilde{T}^{j}\right]=\tilde{f}_{k}^{i j} \widetilde{T}^{k}, \quad\left[T_{i}, \widetilde{T}^{j}\right]=f_{k i}^{j} \widetilde{T}^{k}+\tilde{f}_{i}^{j k} T_{k} .
$$

For many Drinfel'd doubles $\mathscr{D}=(\mathscr{G} \mid \widetilde{\mathscr{G}})$ several Manin triples may exist. Suppose that we have sigma model on $\mathscr{N} \times \mathscr{G}$ and the Drinfel'd double is formed by another pair of subgroups $\widehat{\mathscr{G}}$ and $\overline{\mathscr{G}}$ corresponding to Manin triple $\hat{\mathfrak{g}} \bowtie \overline{\mathfrak{g}}$. Then we can apply the full framework of Poisson-Lie T-plurality [18, 19] and find backgrounds for sigma models on $\mathscr{N} \times \widehat{\mathscr{G}}$ and $\mathscr{N} \times \overline{\mathscr{G}}$. Relation between groups $\mathscr{G}, \widetilde{\mathscr{G}}$ and $\widehat{\mathscr{G}}, \overline{\mathscr{G}}$ is given by relation between the Manin triples. In terms of group elements it reads

$$
l=g(y) \tilde{h}(\tilde{y})=\widehat{g}(\hat{x}) \bar{h}(\bar{x}), \quad l \in \mathscr{D}, g \in \mathscr{G}, \tilde{h} \in \tilde{\mathscr{G}}, \widehat{g} \in \widehat{\mathscr{G}}, \bar{h} \in \overline{\mathscr{G}} .
$$

The mutually dual bases $\widehat{T}_{a} \in \hat{\mathfrak{g}}, \bar{T}^{a} \in \hat{\mathfrak{g}}, a=1, \ldots, d$, satisfying

$$
\begin{aligned}
\left\langle\widehat{T}_{a}, \widehat{T}_{b}\right\rangle & =0, & \left\langle\bar{T}^{a}, \bar{T}^{b}\right\rangle & =0, & \left\langle\widehat{T}_{a}, \bar{T}^{b}\right\rangle & =\delta_{a}^{b}, \\
{\left[\widehat{T}_{i}, \widehat{T}_{j}\right] } & =\hat{f}_{i j}^{k} \widehat{T}_{k}, & {\left[\bar{T}^{i}, \bar{T}^{j}\right] } & =\bar{f}_{k}^{i j} \bar{T}^{k}, & {\left[\widehat{T}_{i}, \bar{T}^{j}\right] } & =\hat{f}_{k i}^{j} \bar{T}^{k}+\bar{f}_{i}^{j k} \widehat{T}_{k}
\end{aligned}
$$

then must be related to $T_{a} \in \mathfrak{g}$ and $\widetilde{T}^{a} \in \tilde{\mathfrak{g}}$ by linear transformation

$$
\left(\begin{array}{l}
\widehat{T} \\
\bar{T}
\end{array}\right)=C \cdot\left(\begin{array}{l}
T \\
\widetilde{T}
\end{array}\right)
$$

where $C$ is an invertible $2 d \times 2 d$ matrix. Given the structure constants $F_{i j}^{k}$ of $\mathfrak{d}=\mathfrak{g} \bowtie \tilde{\mathfrak{g}}$ and $\widehat{F}_{i j}^{k}$ of $\mathfrak{d}=\hat{\mathfrak{g}} \bowtie \overline{\mathfrak{g}}$, the matrix $C$ has to satisfy equation

$$
C_{a}^{p} C_{b}^{q} F_{p q}^{r}=\widehat{F}_{a b}^{c} C_{c}^{r} .
$$

To preserve the bilinear form $\langle.,$.$\rangle and thus (2.4) and (2.6), C$ also has to satisfy

$$
C_{a}^{p} C_{b}^{q}\left(D_{0}\right)_{p q}=\left(D_{0}\right)_{a b}
$$

where $\left(D_{0}\right)_{a b}$ are components of matrix $D_{0}$ that can be written in block form as

$$
D_{0}=\left(\begin{array}{ll}
\mathbf{0}_{d} & \mathbf{1}_{d} \\
\mathbf{1}_{d} & \mathbf{0}_{d}
\end{array}\right) .
$$


In other words, $C$ is an element of $O(d, d)$ but, unlike the case of Abelian T-duality, not every element of $O(d, d)$ is allowed in (2.8).

For the following formulas it will be convenient to introduce $d \times d$ matrices $P, Q, R, S$ as

$$
\left(\begin{array}{l}
T \\
\widetilde{T}
\end{array}\right)=C^{-1} \cdot\left(\begin{array}{c}
\widehat{T} \\
\bar{T}
\end{array}\right)=\left(\begin{array}{ll}
P & Q \\
R & S
\end{array}\right) \cdot\left(\begin{array}{c}
\widehat{T} \\
\bar{T}
\end{array}\right)
$$

and extend these to $(n+d) \times(n+d)$ matrices

$$
\mathcal{P}=\left(\begin{array}{cc}
\mathbf{1}_{n} & 0 \\
0 & P
\end{array}\right), \quad \mathcal{Q}=\left(\begin{array}{cc}
\mathbf{0}_{n} & 0 \\
0 & Q
\end{array}\right), \quad \mathcal{R}=\left(\begin{array}{cc}
\mathbf{0}_{n} & 0 \\
0 & R
\end{array}\right), \quad \mathcal{S}=\left(\begin{array}{cc}
\mathbf{1}_{n} & 0 \\
0 & S
\end{array}\right)
$$

to accommodate the spectator fields. It is also advantageous to introduce block form of $E(s)$ as

$$
E(s)=\left(\begin{array}{cc}
E_{\alpha \beta}(s) & E_{\alpha b}(s) \\
E_{a \beta}(s) & E_{a b}(s)
\end{array}\right), \quad \alpha, \beta=1, \ldots, n, \quad a, b=1, \ldots, d .
$$

The sigma model on $\mathscr{N} \times \widehat{\mathscr{G}}$ related to (2.3) via Poisson-Lie T-plurality is given by tensor

$$
\widehat{\mathcal{F}}(s, \hat{x})=\widehat{\mathcal{E}}(\hat{x}) \cdot \widehat{E}(s, \hat{x}) \cdot \widehat{\mathcal{E}}^{T}(\hat{x}), \quad \widehat{\mathcal{E}}(\hat{x})=\left(\begin{array}{cc}
\mathbf{1}_{n} & 0 \\
0 & \widehat{e}(\hat{x})
\end{array}\right)
$$

where $\widehat{e}(\hat{x})$ is $d \times d$ matrix of components of right-invariant Maurer-Cartan form $(d \hat{g}) \hat{g}^{-1}$ on $\widehat{\mathscr{G}}$,

$$
\begin{aligned}
\widehat{E}(s, \hat{x}) & =\left(\mathbf{1}_{n+d}+\widehat{E}(s) \cdot \widehat{\Pi}(\hat{x})\right)^{-1} \cdot \widehat{E}(s), \\
\widehat{\Pi}(\hat{x}) & =\left(\begin{array}{cc}
\mathbf{0}_{n} & 0 \\
0 & \widehat{b}(\hat{x}) \cdot \widehat{a}^{-1}(\hat{x})
\end{array}\right),
\end{aligned}
$$

and matrices $\widehat{b}(\hat{x})$ and $\widehat{a}(\hat{x})$ are submatrices of the adjoint representation

$$
\operatorname{ad}_{\hat{g}^{-1}}(\bar{T})=\widehat{b}(\hat{x}) \cdot \widehat{T}+\widehat{a}^{-1}(\hat{x}) \cdot \bar{T} .
$$

The matrix $\widehat{E}(s)$ is obtained from $E(s)$ in $(2.3)$ by formula

$$
\widehat{E}(s)=(\mathcal{P}+E(s) \cdot \mathcal{R})^{-1} \cdot(\mathcal{Q}+E(s) \cdot \mathcal{S})
$$

so it is necessary that ${ }^{2}$

$$
\operatorname{det}(\mathcal{P}+E(s) \cdot \mathcal{R}) \neq 0 .
$$

Analogously we get tensor dual to $(2.13)$ on $\mathscr{N} \times \overline{\mathscr{G}}$.

Formulas (2.13)-(2.15) reduce to those for full Poisson-Lie duality if we choose $P=$ $S=\mathbf{0}_{d}$ and $Q=R=\mathbf{1}_{d}$. Furthermore, for a semi-Abelian Drinfel'd double the wellknown Buscher rules for (non-)Abelian T-duality are restored. If there are no spectators the plurality is called atomic.

\footnotetext{
${ }^{2}$ For regular $\widehat{E}(s)$ formula $(2.14)$ simplifies to $\left(\widehat{E}^{-1}(s)+\widehat{\Pi}(\hat{x})\right)^{-1}$ and $\operatorname{det}(\mathcal{Q}+E(s) \cdot \mathcal{S}) \neq 0$ is also needed.
} 


\subsection{Generalized Supergravity Equations and transformation of dilaton}

Main goal of this paper is to verify whether backgrounds obtained by Poisson-Lie Tpluralities satisfy Generalized Supergravity Equations of Motion [11, 12, 30, 31]. They can be written in different forms. We adopt convention used in [14] so the equations read ${ }^{3}$

$$
\begin{aligned}
& 0=R_{\mu \nu}-\frac{1}{4} H_{\mu \rho \sigma} H_{\nu}^{\rho \sigma}+\nabla_{\mu} X_{\nu}+\nabla_{\nu} X_{\mu}, \\
& 0=-\frac{1}{2} \nabla^{\rho} H_{\rho \mu \nu}+X^{\rho} H_{\rho \mu \nu}+\nabla_{\mu} X_{\nu}-\nabla_{\nu} X_{\mu}, \\
& 0=R-\frac{1}{12} H_{\rho \sigma \tau} H^{\rho \sigma \tau}+4 \nabla_{\mu} X^{\mu}-4 X_{\mu} X^{\mu}
\end{aligned}
$$

where

$$
H_{\rho \mu \nu}=\partial_{\rho} \mathcal{B}_{\mu \nu}+\partial_{\mu} \mathcal{B}_{\nu \rho}+\partial_{\nu} \mathcal{B}_{\rho \mu}
$$

$\nabla_{\mu}$ are covariant derivatives with respect to metric $\mathcal{G}$,

$$
X_{\mu}=\partial_{\mu} \Phi+\mathcal{J}^{\nu} \mathcal{F}_{\nu \mu}
$$

$\Phi$ is the dilaton and vector field $\mathcal{J}$ will be defined bellow. For vanishing $\mathcal{J}$ the usual beta function equations are recovered.

Formula for transformation of dilaton under Poisson-Lie T-plurality was given in [19] as

$$
\widehat{\Phi}(\hat{x})=\Phi^{0}(y)-\frac{1}{2} \ln |\operatorname{det}[(N+\widehat{\Pi}(\hat{x}) M) \widehat{a}(\hat{x})]|
$$

where $y$ represent coordinates of $\mathscr{G}$,

$$
\Phi^{0}(y)=\Phi(y)+\frac{1}{2} \ln |\operatorname{det}[(\mathbf{1}+\Pi(y) E(s)) a(y)]|,
$$

$\Phi(y)$ is the dilaton of the initial model and

$$
M=\mathcal{S}^{T} E(s)-\mathcal{Q}^{T}, \quad N=\mathcal{P}^{T}-\mathcal{R}^{T} E(s) .
$$

Problem with the formula (2.20) is that in general it is non-local in the sense that $y=y(\hat{x}, \bar{x})$ obtained by decompositions of Drinfel'd double elements

$$
l=g(y) \tilde{h}(\tilde{y})=\widehat{g}(\hat{x}) \bar{h}(\bar{x}), \quad l \in \mathscr{D}, g \in \mathscr{G}, \tilde{h} \in \tilde{\mathscr{G}}, \widehat{g} \in \widehat{\mathscr{G}}, \bar{h} \in \overline{\mathscr{G}} .
$$

This is the cause of the puzzle mentioned in [23], namely that $\widehat{\Phi}$ in formula (2.20) for dilaton on $\widehat{\mathscr{G}}$ depends not only on coordinates $\hat{x}$ of the group $\widehat{\mathscr{G}}$ but also on coordinates $\bar{x}$ of $\overline{\mathscr{G}}$.

For coordinates $y$ depending linearly on $\hat{x}$ and $\bar{x}$

$$
y^{k}(\hat{x}, \bar{x})=\hat{d}_{m}^{k} \hat{x}^{m}+\bar{d}^{k m} \bar{x}_{m},
$$

which holds for all cases bellow, the problem can be solved in the following way. We set

$$
\widehat{\Phi}^{0}(y)=\Phi^{0}\left(\hat{d}_{m}^{k} \hat{x}^{m}\right)
$$

\footnotetext{
${ }^{3}$ We consider here only bosonic fields in the backgrounds.
} 
and vectors $\widehat{\mathcal{J}}$ for backgrounds on $\mathscr{N} \times \widehat{\mathscr{G}}$

$$
\begin{aligned}
\widehat{\mathcal{J}}^{\alpha} & =0, & \alpha & =1, \ldots, \operatorname{dim} \mathscr{N} \\
\widehat{\mathcal{J}}^{\operatorname{dim} \mathscr{N}+m} & =\left(\frac{1}{2} \bar{f}_{b}^{a b}-\frac{\partial \Phi^{0}}{\partial y^{k}} \bar{d}^{k a}\right) \widehat{V}_{a}^{m}, & a, b, k, m & =1, \ldots, \operatorname{dim} \mathscr{G} .
\end{aligned}
$$

For the dual model on $\mathscr{N} \times \overline{\mathscr{G}}$

$$
\begin{aligned}
\bar{\Phi}^{0}(y) & =\Phi^{0}\left(\bar{d}^{k m} \bar{x}_{m}\right), \\
\overline{\mathcal{J}}^{\alpha}=0, \quad \overline{\mathcal{J}}^{\operatorname{dim} \mathscr{N}+m} & =\left(\frac{1}{2} \hat{f}_{a b}{ }^{b}-\frac{\partial \Phi^{0}}{\partial y^{k}} \hat{d}_{a}^{k}\right) \bar{V}^{a, m},
\end{aligned}
$$

where $\widehat{V}_{a}, \bar{V}^{a}$ are left-invariant fields of the groups $\widehat{\mathscr{G}}, \overline{\mathscr{G}}$ and $\hat{f}_{b a}{ }^{c}$ and $\bar{f}^{b a}{ }_{c}$ are structure constants of their Lie algebras. Note that in case of Poisson-Lie T-plurality linear combinations of left-invariant vector fields need not be Killing vectors of plural backgrounds as they satisfy condition [18] of pluralizability

$$
\left(\mathfrak{L}_{\widehat{V}_{a}} \widehat{\mathcal{F}}\right)_{\mu \nu}=\widehat{\mathcal{F}}_{\mu \kappa} \widehat{V}_{b}^{\kappa} \bar{f}_{a}^{b c} \widehat{V}_{c}^{\lambda} \widehat{\mathcal{F}}_{\lambda \nu}
$$

with generally nonvanishing right-hand side. Nevertheless, it turns out that all nontrivial vectors $\widehat{\mathcal{J}}$ and $\overline{\mathcal{J}}$ found below are Killing vectors of corresponding backgrounds.

For non-Abelian T-duality, authors of [24, 32] calculate components $\mathcal{J}^{\mu}$ of vector $\mathcal{J}$ using

$$
(\operatorname{Div} \Theta)^{\mu}=D_{\nu} \Theta^{\nu \mu}
$$

where $^{4}$

$$
\Theta^{\mu \nu}=-\left((\mathcal{G}+\mathcal{B})^{-1} \cdot \mathcal{B} \cdot(\mathcal{G}-\mathcal{B})^{-1}\right)^{\mu \nu},
$$

$\mathcal{G}$ and $\mathcal{B}$ are symmetric and antisymmetric part of tensor $\mathcal{F}$ and $D_{\nu}$ is covariant derivative with respect to

$$
G_{\mu \nu}=\left(\mathcal{G}-\mathcal{B} \cdot \mathcal{G}^{-1} \cdot \mathcal{B}\right)_{\mu \nu}
$$

Unfortunately, as we shall show on many examples below, this formula does not work in general for Poisson-Lie T-plurality.

\section{Poisson-Lie T-plurality of flat background}

\subsection{Bianchi III cosmology}

First we shall study Poisson-Lie T-pluralities that follow from the Bianchi $I I I$ invariance of Minkowski metric. Consider six-dimensional semi-Abelian Drinfel'd double ${ }^{5} \mathscr{D}=\left(\mathscr{B}_{I I I} \mid \mathscr{A}\right)$ whose Lie algebra $\mathfrak{d}=\mathfrak{b}_{I I I} \bowtie \mathfrak{a}$ is spanned by basis $\left(T_{1}, T_{2}, T_{3}, \widetilde{T}^{1}, \widetilde{T}^{2}, \widetilde{T}^{3}\right)$ with nonvanishing commutation relations

$$
\left[T_{1}, T_{3}\right]=-T_{3}, \quad\left[T_{1}, \widetilde{T}^{3}\right]=\widetilde{T}^{3}, \quad\left[T_{3}, \widetilde{T}^{3}\right]=-\widetilde{T}^{1} .
$$

\footnotetext{
${ }^{4}$ Note that $\Theta$ is the antisymmetric part of $\mathcal{F}^{-1}(s, x)$.

${ }^{5} \mathrm{By} \mathscr{B}_{I I I}$ and $\mathfrak{b}_{I I I}$ we denote the group Bianchi $I I I$ resp. its Lie algebra and by $\mathscr{A}$ and $\mathfrak{a}$ we denote three dimensional Abelian group resp. its Lie algebra. Similar notation will be used in the following sections.
} 
The group $\mathscr{B}_{I I I}$ is not semisimple and trace of its structure constants does not vanish. Lie algebra $\mathfrak{d}$ of this Drinfel'd double admits several other decompositions into Manin triples found in [22].

In adapted coordinates the Bianchi $I I I$ flat cosmology is given by metric ${ }^{6}$

$$
\mathcal{F}\left(t, y_{1}\right)=\left(\begin{array}{cccc}
-1 & 0 & 0 & 0 \\
0 & t^{2} & 0 & 0 \\
0 & 0 & 1 & 0 \\
0 & 0 & 0 & e^{-2 y_{1}} t^{2}
\end{array}\right)
$$

The corresponding matrix $E(s)$ can be found by setting $y_{1}=0$ in $\mathcal{F}$. The background is invariant with respect to the action of Bianchi $I I I$ group generated by left-invariant vector fields

$$
V_{1}=\partial_{y_{1}}+y_{3} \partial_{y_{3}}, \quad V_{2}=\partial_{y_{2}}, \quad V_{3}=\partial_{y_{3}}
$$

The metric is flat and there is no torsion so the equations (2.17)-(2.19) are satisfied if we choose dilaton $\Phi(y)=0$ and vector $\mathcal{J}=0$. This background was mentioned in [33] where the authors noted that its non-Abelian dual does not satisfy standard beta function equations.

The equation (2.21) implies $\Phi^{(0)}(y)=\frac{y_{1}}{2}$ and formula (2.20) for new dilatons then simplifies to

$$
\widehat{\Phi}(\hat{x}, \bar{x})=\frac{y_{1}(\hat{x}, \bar{x})}{2}-\frac{1}{2} \ln |\operatorname{det}[(N+\widehat{\Pi}(\hat{x}) M) \widehat{a}(\hat{x})]| .
$$

Hence, for every $\hat{\mathfrak{g}} \bowtie \overline{\mathfrak{g}}$ it is necessary to express $y_{1}$ in terms of $\hat{x}$ and $\bar{x}$ from (2.22).

\subsubsection{Transformation of $\mathfrak{b}_{I I I} \bowtie \mathfrak{a}$ to $\mathfrak{b}_{I I I} \bowtie \mathfrak{b}_{I I}$ and to its dual}

As was found in [22], the algebra of the Drinfel'd double $\mathscr{D}=\left(\mathscr{B}_{I I I} \mid \mathscr{A}\right)$ can be decomposed into Manin triple $\mathfrak{d}=\mathfrak{b}_{I I I} \bowtie \mathfrak{b}_{I I}$ spanned by basis $\left(\widehat{T}_{1}, \widehat{T}_{2}, \widehat{T}_{3}, \bar{T}^{1}, \bar{T}^{2}, \bar{T}^{3}\right)$ with non-trivial algebraic relations

$$
\begin{array}{rlrl}
{\left[\widehat{T}_{1}, \widehat{T}_{2}\right]=-\widehat{T}_{2},} & {\left[\widehat{T}_{1}, \bar{T}^{2}\right]=\left(\widehat{T}_{3}+\bar{T}^{2}\right),} & {\left[\widehat{T}_{1}, \bar{T}^{3}\right]=-\widehat{T}_{2},} \\
{\left[\widehat{T}_{2}, \bar{T}^{2}\right]=-\bar{T}^{1},} & {\left[\bar{T}^{2}, \bar{T}^{3}\right]=\bar{T}^{1}}
\end{array}
$$

Left-invariant fields of $\widehat{\mathscr{G}}=\mathscr{B}_{I I I}$ and $\overline{\mathscr{G}}=\mathscr{B}_{I I}$ are

$$
\begin{array}{lll}
\widehat{V}_{1}=\partial_{\hat{x}_{1}}+\hat{x}_{2} \partial_{\hat{x}_{2}}, & \widehat{V}_{2}=\partial_{\hat{x}_{2},} & \widehat{V}_{3}=\partial_{\hat{x}_{3}}, \\
\bar{V}_{1}=\partial_{\bar{x}_{1}}, & \bar{V}_{2}=-\bar{x}_{3} \partial_{\bar{x}_{1}}+\partial_{\bar{x}_{2}}, & \bar{V}_{3}=\partial_{\bar{x}_{3}} .
\end{array}
$$

There is rather large number of solutions of equations (2.9) and (2.10) giving linear mappings (2.8) between generators of $\mathfrak{b}_{I I I} \bowtie \mathfrak{a}$ and $\mathfrak{b}_{I I I} \bowtie \mathfrak{b}_{I I}$ (cf. the table of Poisson-Lie identities of $\mathfrak{b}_{I I I} \bowtie \mathfrak{a}$ in [16]). However, up to a change of coordinates and gauge shift of the $\widehat{\mathcal{B}}$ field, all the resulting plural backgrounds $\widehat{\mathcal{F}}$ on $\widehat{\mathscr{G}}$ are equivalent. Since the parameters

\footnotetext{
${ }^{6}$ For typographic reasons from now on we shall use subscripts to label group coordinates.
} 
coming from mappings $C$ can be eliminated in plural backgrounds by transformations of coordinates or gauge shifts, it is sufficient to consider matrix

$$
C_{1}=\left(\begin{array}{cccccc}
1 & 0 & 0 & 0 & 0 & 0 \\
0 & 0 & 1 & 0 & 0 & 0 \\
0 & 1 & 0 & 0 & 0 & 0 \\
0 & 0 & 0 & 1 & 0 & 0 \\
0 & -1 & 0 & 0 & 0 & 1 \\
0 & 0 & 1 & 0 & 1 & 0
\end{array}\right),
$$

which gives rise to tensor $\widehat{\mathcal{F}}$ on $\widehat{\mathscr{G}}$ calculated from (2.13), (2.14) as

$$
\widehat{\mathcal{F}}\left(t, \hat{x}_{1}\right)=\left(\begin{array}{cccc}
-1 & 0 & 0 & 0 \\
0 & t^{2} & 0 & 0 \\
0 & 0 & \frac{t^{2}}{e^{2 \hat{x}_{1}}+t^{2}} & -\frac{t^{2}}{e^{2 \hat{x}_{1}}+t^{2}} \\
0 & 0 & \frac{t^{2}}{e^{2 \hat{x}_{1}}+t^{2}} & \frac{e^{2 \hat{x}_{1}}}{e^{2 \hat{x}_{1}}+t^{2}}
\end{array}\right) .
$$

The metric is not flat but its scalar curvature vanishes. It is possible to find coordinate transformation that brings the metric to the Brinkmann form of a plane parallel wave

$$
d s^{2}=\frac{\left(2 u^{2}-1\right) z_{3}^{2}-3 z_{4}^{2}}{\left(1+u^{2}\right)^{2}} d u^{2}+2 d u d v+d z_{3}^{2}+d z_{4}^{2} .
$$

The background is accompanied by a non-trivial torsion

$$
\widehat{H}=\frac{2}{1+u^{2}} d u \wedge d z_{3} \wedge d z_{4} .
$$

This background was repeatedly found in [35-37] as non-Abelian T-dual of flat metric.

Let us now focus on the dilaton. Change of Manin triples given by mapping (3.6) induces transformation of coordinates of the Drinfel'd double. From (2.22) we find that

$$
\begin{aligned}
& y_{1}=\hat{x}_{1}, \quad y_{2}=\hat{x}_{3}-\bar{x}_{2}, \quad y_{3}=\hat{x}_{2}+\bar{x}_{3}, \\
& \tilde{y}_{1}=\bar{x}_{1}-\bar{x}_{2} \bar{x}_{3}, \quad \tilde{y}_{2}=\bar{x}_{3}, \quad \tilde{y}_{3}=\bar{x}_{2} .
\end{aligned}
$$

As $\Phi^{(0)}(y)=\frac{y_{1}}{2}$, we need only $y_{1}$ and that gives us $\Phi^{(0)}=\frac{\hat{x}_{1}}{2}$. From the formula (2.20) we obtain transformed dilaton

$$
\widehat{\Phi}\left(t, \hat{x}_{1}\right)=-\frac{1}{2} \ln \left(t^{2} e^{-2 \hat{x}_{1}}+1\right) .
$$

Vector $\widehat{\mathcal{J}}$ given by both (2.23) and (2.26) vanishes, Generalized Supergravity Equations become standard beta function equations, and one can check that they are satisfied.

For sigma model on $\overline{\mathscr{G}}$ different $C$ matrices give different backgrounds. For instance, using

$$
C_{2}=D_{0} \cdot C_{1}=\left(\begin{array}{cccccc}
0 & 0 & 0 & 1 & 0 & 0 \\
0 & -1 & 0 & 0 & 0 & 1 \\
0 & 0 & 1 & 0 & 1 & 0 \\
1 & 0 & 0 & 0 & 0 & 0 \\
0 & 0 & 1 & 0 & 0 & 0 \\
0 & 1 & 0 & 0 & 0 & 0
\end{array}\right)
$$


we obtain tensor

$$
\overline{\mathcal{F}}\left(t, \bar{x}_{2}\right)=\left(\begin{array}{cccc}
-1 & 0 & 0 & 0 \\
0 & \frac{t^{2}}{t^{4}+\bar{x}_{2}^{2}} & -\frac{\bar{x}_{2}}{t^{4}+\bar{x}_{2}^{2}} & 0 \\
0 & \frac{\bar{x}_{2}}{t^{4}+\bar{x}_{2}^{2}} & \frac{t^{2}}{t^{4}+\bar{x}_{2}^{2}} & 1 \\
0 & 0 & -1 & 1
\end{array}\right)
$$

with non-trivial curvature and torsion. To find the dilaton and vector $\overline{\mathcal{J}}$ we express $y_{1}$ from $(2.22)$ as $y_{1}=\hat{x}_{1}$. Dilaton

$$
\bar{\Phi}\left(t, \bar{x}_{2}\right)=-\frac{1}{2} \ln \left(t^{4}+\bar{x}_{2}^{2}\right)
$$

found by Poisson-Lie T-plurality generated by $C_{2}=D_{0} \cdot C_{1}$ transforming $\mathfrak{b}_{I I I} \bowtie \mathfrak{a}$ to $\mathfrak{b}_{I I} \bowtie \mathfrak{b}_{I I I}$ satisfies Generalized Supergravity Equations if the vector

$$
\overline{\mathcal{J}}=(0,-1,0,0)
$$

is calculated via (2.24). Formula (2.26) fails to provide correct $\overline{\mathcal{J}}$ as

$$
D_{\nu} \Theta^{\nu \mu}=\left(0,-\frac{1}{1+t^{2}}, 0,0\right)
$$

Using

$$
C_{3}=\left(\begin{array}{cccccc}
0 & 0 & 0 & -1 & 0 & 0 \\
0 & 0 & 1 & 0 & -1 & 0 \\
0 & 1 & 0 & 0 & 0 & 1 \\
-1 & 0 & 0 & 0 & 0 & 0 \\
0 & 0 & 0 & 0 & 0 & 1 \\
0 & 0 & 0 & 0 & 1 & 0
\end{array}\right)
$$

we get torsionless background

$$
\overline{\mathcal{F}}\left(t, \bar{x}_{2}\right)=\left(\begin{array}{cccc}
-1 & 0 & 0 & 0 \\
0 & \frac{1}{\left(\bar{x}_{2}^{2}+1\right) t^{2}} & -\frac{\bar{x}_{2}}{\bar{x}_{2}^{2}+1} & 0 \\
0 & \frac{\bar{x}_{2}}{\bar{x}_{2}^{2}+1} & \frac{t^{2}}{\bar{x}_{2}^{2}+1} & 1 \\
0 & 0 & -1 & 1
\end{array}\right) .
$$

From (2.22) we find that $y_{1}=-\hat{x}_{1}$ and formulas (2.20) and (2.24) give

$$
\bar{\Phi}\left(t, \bar{x}_{2}\right)=-\frac{1}{2} \ln \left(t^{2}\left(1+\bar{x}_{2}^{2}\right)\right), \quad \overline{\mathcal{J}}=(0,0,0,0) .
$$

The standard beta function equations are thus satisfied. Since $D_{\nu} \Theta^{\nu \mu}=\left(0,-\frac{t^{2}}{1+t^{2}}, 0,0\right)$, we see that formula (2.26) is again not applicable. 


\subsubsection{Transformation of $\mathfrak{b}_{I I I} \bowtie \mathfrak{a}$ to $\mathfrak{b}_{I I I} \bowtie \mathfrak{b}_{I I I i i i}$ and to its dual}

Lie algebra $\mathfrak{d}=\mathfrak{b}_{I I I} \bowtie \mathfrak{b}_{\text {IIIiii }}$ is spanned by $\left(\widehat{T}_{1}, \widehat{T}_{2}, \widehat{T}_{3}, \bar{T}^{1}, \bar{T}^{2}, \bar{T}^{3}\right)$ with non-trivial algebraic relations

$$
\begin{aligned}
& {\left[\widehat{T}_{1}, \widehat{T}_{2}\right]=-4 \widehat{T}_{2}, \quad\left[\widehat{T}_{1}, \bar{T}^{1}\right]=2 \widehat{T}_{2}, \quad\left[\widehat{T}_{1}, \bar{T}^{2}\right]=\left(-2 \widehat{T}_{1}+4 \bar{T}^{2}\right),} \\
& {\left[\widehat{T}_{2}, \bar{T}^{2}\right]=-4 \bar{T}^{1}, \quad\left[\bar{T}^{1}, \bar{T}^{2}\right]=2 \bar{T}^{1} .}
\end{aligned}
$$

The left-invariant fields of $\widehat{\mathscr{G}}$ and $\overline{\mathscr{G}}$ are

$$
\begin{array}{lll}
\widehat{V}_{1}=\partial_{\hat{x}_{1}}+4 \hat{x}_{2} \partial_{\hat{x}_{2},} & \widehat{V}_{2}=\partial_{\hat{x}_{2},} & \widehat{V}_{3}=\partial_{\hat{x}_{3}}, \\
\bar{V}_{1}=e^{-2 \bar{x}_{2}} \partial_{\bar{x}_{1}}, & \bar{V}_{2}=\partial_{\bar{x}_{2}}, & \bar{V}_{3}=\partial_{\bar{x}_{3}} .
\end{array}
$$

There is again rather large number of linear mappings (2.8) between $\mathfrak{b}_{I I I} \bowtie \mathfrak{a}$ and $\mathfrak{b}_{I I I} \bowtie$ $\mathfrak{b}_{\text {IIIiii }}$ depending on many parameters. The backgrounds obtained by plurality are quite complicated, hence, we shall restrict to two simple ones.

First of them is given by matrix

$$
C_{1}=\left(\begin{array}{cccccc}
-4 & 0 & 0 & 0 & 0 & 0 \\
0 & 0 & 0 & 0 & 0 & 1 \\
0 & 1 & 0 & 0 & 0 & 0 \\
0 & 0 & 0 & -\frac{1}{4} & 0 & -\frac{1}{2} \\
-2 & 0 & 1 & 0 & 0 & 0 \\
0 & 0 & 0 & 0 & 1 & 0
\end{array}\right)
$$

for which tensor $\widehat{\mathcal{F}}$ has the form

$$
\widehat{\mathcal{F}}\left(t, \hat{x}_{1}\right)=\left(\begin{array}{cccc}
-1 & 0 & 0 & 0 \\
0 & \frac{16 e^{8 \hat{x}_{1} t^{2}}}{4+e^{8 \hat{x}_{1}}} & -\frac{8}{4+e^{8 \hat{x}_{1}}} & 0 \\
0 & \frac{8}{4+e^{8 \hat{x}_{1}}} & \frac{1}{\left(4+e^{8 \hat{x}_{1}}\right) t^{2}} & 0 \\
0 & 0 & 0 & 1
\end{array}\right) .
$$

The background is curved and torsionless. From the change of decompositions (2.22) we express $y_{1}$ as

$$
y_{1}=-4 \hat{x}_{1}-2 \bar{x}_{2}
$$

Dilaton

$$
\widehat{\Phi}\left(t, \hat{x}_{1}\right)=-\frac{1}{2} \ln \left(\frac{t^{2}}{4}\left(4+e^{8 \hat{x}_{1}}\right)\right)
$$

satisfies Generalized Supergravity Equations with $\widehat{\mathcal{J}}=(0,0,0,0)$ but $\operatorname{Div} \Theta=(0,0,-2,0)$.

Torsionless background

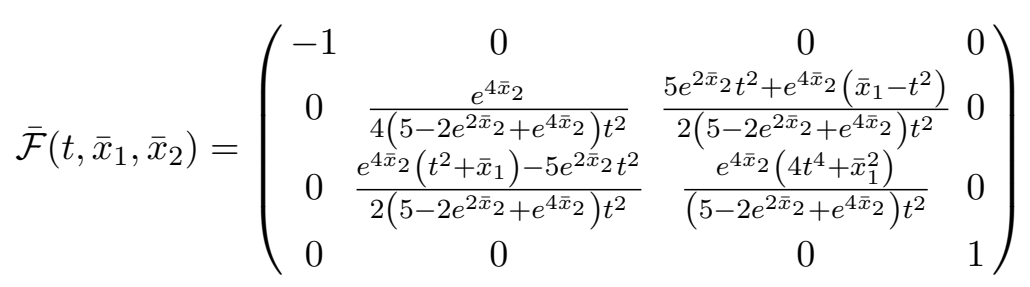


is obtained by Poisson-Lie T-plurality given by $D_{0} \cdot C_{1}$. Transforming $\mathfrak{b}_{\text {III }} \bowtie \mathfrak{a}$ to $\mathfrak{b}_{\text {IIIiii }} \bowtie$ $\mathfrak{b}_{I I I}$ we get dilaton

$$
\bar{\Phi}\left(t, \bar{x}_{2}\right)=-\frac{1}{2} \ln \left(t^{2}\left(-2 e^{2 \bar{x}_{2}}+e^{4 \bar{x}_{2}}+5\right)\right)
$$

that satisfies beta function equations, i.e. Generalized Supergravity Equations with $\overline{\mathcal{J}}=$ $(0,0,0,0)$. That disagrees with formula $(2.26)$ giving $\operatorname{Div} \Theta=\left(0,-4 e^{-2 \bar{x}_{2}}, 0,0\right)$.

Another Poisson-Lie T-plurality is given by the matrix

$$
C_{2}=\left(\begin{array}{cccccc}
4 & 0 & 0 & 0 & 0 & 0 \\
0 & 0 & 2 & 0 & 0 & 0 \\
0 & 1 & 0 & 0 & 0 & 0 \\
0 & 0 & -1 & \frac{1}{4} & 0 & 0 \\
2 & 0 & 0 & 0 & 0 & \frac{1}{2} \\
0 & 0 & 0 & 0 & 1 & 0
\end{array}\right)
$$

that leads to background

$$
\widehat{\mathcal{F}}\left(t, \hat{x}_{1}\right)=\left(\begin{array}{cccc}
-1 & 0 & 0 & 0 \\
0 & \frac{16 e^{8 \hat{x}_{1}} t^{2}}{16 t^{4}+e^{8 \hat{x}_{1}}} & -\frac{32 t^{4}}{16 t^{4}+e^{8 \widehat{x}_{1}}} & 0 \\
0 & \frac{32 t^{4}}{16 t^{4}+e^{8 \hat{x}_{1}}} & \frac{4 t^{2}}{16 t^{4}+e^{8 \hat{x}_{1}}} & 0 \\
0 & 0 & 0 & 1
\end{array}\right)
$$

with nontrivial torsion. For $C_{2}$ we find that

$$
y_{1}=4 \hat{x}_{1}+2 \bar{x}_{2}, \quad \Phi^{(0)}=2 \hat{x}_{1},
$$

and the background supported by dilaton

$$
\widehat{\Phi}\left(t, \hat{x}_{1}\right)=-\frac{1}{2} \ln \left(2 t^{4} e^{-8 \hat{x}_{1}}+\frac{1}{8}\right)
$$

satisfies Generalized Supergravity Equations with $\widehat{\mathcal{J}}=(0,0,-2,0)$ obtained by both $(2.23)$ and (2.26). Background obtained by the Poisson-Lie T-plurality given by $D_{0} \cdot C_{2}$ transforming $\mathfrak{b}_{I I I} \bowtie \mathfrak{a}$ to $\mathfrak{b}_{I I I i i i} \bowtie \mathfrak{b}_{I I I}$ is

$$
\overline{\mathcal{F}}\left(t, \bar{x}_{1}, \bar{x}_{2}\right)=\left(\begin{array}{cccc}
-1 & 0 & 0 & 0 \\
0 & \frac{e^{4 \bar{x}_{2}} t^{2}}{16 t^{4}-2 e^{2 \bar{x}_{2}}+e^{4 \bar{x}_{2}+1}} & \frac{e^{2 \bar{x}_{2}}\left(16 t^{4}+e^{2 \bar{x}_{2}}\left(4 t^{2} \bar{x}_{1}-1\right)+1\right)}{2\left(16 t^{4}-2 e^{\left.2 \bar{x}_{2}+e^{4} \bar{x}_{2}+1\right)}\right.} & 0 \\
0 & \frac{e^{2 \bar{x}_{2}}\left(-16 t^{4}+e^{2 \bar{x}_{2}}\left(4 \bar{x}_{1} t^{2}+1\right)-1\right)}{2\left(16 t^{4}-2 e^{\left.2 \bar{x}_{2}+e^{4} \bar{x}_{2}+1\right)}\right.} & \frac{4 e^{4 \bar{x}_{2}} t^{2}\left(\bar{x}_{1}^{2}+1\right)}{16 t^{4}-2 e^{2 \bar{x}_{2}}+e^{4 \bar{x}_{2}+1}} & 0 \\
0 & 0 & 0 & 1
\end{array}\right) .
$$

Dilaton

$$
\bar{\Phi}\left(t, \bar{x}_{2}\right)=-\frac{1}{2} \ln \left(\frac{1}{2} e^{-4 \bar{x}_{2}}\left(16 t^{4}-2 e^{2 \bar{x}_{2}}+e^{4 \bar{x}_{2}}+1\right)\right)
$$

calculated via formula (3.4) and background (3.7) satisfy Generalized Supergravity Equations with non-constant vector

$$
\overline{\mathcal{J}}=\left(0,-4 e^{-2 \bar{x}_{2}}, 0,0\right)
$$


obtained from both (2.23) and (2.26). Although it is not obvious, $\overline{\mathcal{J}}$ is a Killing vector of (3.7). This example shows that components of left-invariant fields need to appear in formulas (2.23), (2.24). Papers [14, 16] dealt only with semi-Abelian Drinfel'd doubles where $\bar{V}^{a, m}=\delta^{a, m}$, vectors $\overline{\mathcal{J}}$ were constant and proportional to the trace of structure constants so the full form of (2.24) was not necessary.

\subsection{Bianchi $V$ cosmology}

Next we shall study Poisson-Lie T-pluralities that follow from the well-known Bianchi $V$ invariance of the flat metric that in adapted coordinates reads

$$
\mathcal{F}\left(t, y_{1}\right)=\left(\begin{array}{cccc}
-1 & 0 & 0 & 0 \\
0 & t^{2} & 0 & 0 \\
0 & 0 & e^{2 y_{1}} t^{2} & 0 \\
0 & 0 & 0 & e^{2 y_{1}} t^{2}
\end{array}\right)
$$

Authors of [7] first noticed that non-Abelian dual of this background is not conformal. Further study of the emerging gravitational-gauge anomaly was carried out in [34].

Metric (3.8) is invariant with respect to the action of Bianchi $V$ group and can be obtained by formula (2.3) if we consider six-dimensional semi-Abelian Drinfel'd double $\mathscr{D}=\left(\mathscr{B}_{V} \mid \mathscr{A}\right)$ and Manin triple $\mathfrak{d}=\mathfrak{b}_{V} \bowtie \mathfrak{a}$ spanned by basis $\left(T_{1}, T_{2}, T_{3}, \widetilde{T}^{1}, \widetilde{T}^{2}, \widetilde{T}^{3}\right)$ with non-trivial comutation relations

$$
\begin{array}{llll}
{\left[T_{1}, T_{2}\right]} & =T_{2}, & {\left[T_{1}, T_{3}\right]=T_{3},} & {\left[T_{1}, \widetilde{T}^{2}\right]=-\widetilde{T}^{2},} \\
{\left[T_{1}, \widetilde{T}^{3}\right]=-\widetilde{T}^{3},} & {\left[T_{2}, \widetilde{T}^{2}\right]=\widetilde{T}^{1},} & {\left[T_{3}, \widetilde{T}^{3}\right]=\widetilde{T}^{1} .}
\end{array}
$$

The group $\mathscr{B}_{V}$ is not semisimple and trace of its structure constants does not vanish. The algebra $\mathfrak{d}$ allows several other decompositions into Manin triples $\hat{\mathfrak{g}} \bowtie \overline{\mathfrak{g}}$.

Corresponding dilaton can be again chosen $\Phi=0$ and transformation formulas $(2.20),(2.21)$ now give

$$
\widehat{\Phi}(\hat{x}, \bar{x})=-y_{1}(\hat{x}, \bar{x})-\frac{1}{2} \ln |\operatorname{det}[(N+\widehat{\Pi}(\hat{x}) M) \widehat{a}(\hat{x})]|
$$

so it is again necessary to find how $y_{1}$ depends on $\hat{x}, \bar{x}$ using $(2.22)$.

\subsubsection{Transformation of $\mathfrak{b}_{V} \bowtie \mathfrak{a}$ to $\mathfrak{b}_{V I_{-1}} \bowtie \mathfrak{a}$ and to its dual}

Lie algebra $\mathfrak{d}=\mathfrak{b}_{V I_{-1}} \bowtie \mathfrak{a}$ is spanned by $\left(\widehat{T}_{1}, \widehat{T}_{2}, \widehat{T}_{3}, \bar{T}^{1}, \bar{T}^{2}, \bar{T}^{3}\right)$ with algebraic relations

$$
\begin{array}{lll}
{\left[\widehat{T}_{1}, \widehat{T}_{2}\right]=-\widehat{T}_{2},} & {\left[\widehat{T}_{1}, \widehat{T}_{3}\right]=\widehat{T}_{3},} & {\left[\widehat{T}_{1}, \bar{T}^{2}\right]=\bar{T}^{2},} \\
{\left[\widehat{T}_{1}, \bar{T}^{3}\right]=-\bar{T}^{3},} & {\left[\widehat{T}_{2}, \bar{T}^{2}\right]=-\bar{T}^{1},} & {\left[\widehat{T}_{3}, \bar{T}^{3}\right]=\bar{T}^{1} .}
\end{array}
$$

Left-invariant vector fields of $\widehat{\mathscr{G}}$ and $\overline{\mathscr{G}}$ are

$$
\begin{array}{lll}
\widehat{V}_{1}=\partial_{\hat{x}_{1}}+\hat{x}_{2} \partial_{\hat{x}_{2}}-\hat{x}_{3} \partial_{\hat{x}_{3},} & \widehat{V}_{2}=\partial_{\hat{x}_{2}}, & \widehat{V}_{3}=\partial_{\hat{x}_{3}}, \\
\bar{V}_{1}=\partial_{\bar{x}_{1}}, & \bar{V}_{2}=\partial_{\bar{x}_{2}}, & \bar{V}_{3}=\partial_{\bar{x}_{3}} .
\end{array}
$$


Mappings that transform commutation relations of Manin triple $\mathfrak{b}_{V} \bowtie \mathfrak{a}$ to those of $\mathfrak{b}_{V I_{-1}} \bowtie \mathfrak{a}$ are in general given by matrices

$$
C_{1}=\left(\begin{array}{cccccc}
1 & c_{12} & c_{13} & -c_{12} c_{15}-c_{13} c_{16} & c_{15} & c_{16} \\
0 & 0 & 0 & -c_{12} c_{25}-c_{13} c_{26} & c_{25} & c_{26} \\
0 & \frac{c_{26}}{c_{26} c_{65}-c_{25} c_{66}} & \frac{c_{25}}{c_{25} c_{66}-c_{26} c_{65}} & \frac{c_{16} c_{25}-c_{15} c_{26}}{c_{26} c_{65}-c_{25} c_{66}} & 0 & 0 \\
0 & 0 & 0 & 1 & 0 & 0 \\
0 & \frac{c_{66}}{c_{25} c_{66}-c_{26} c_{65}} & \frac{1}{c_{26}-\frac{c_{25} c_{66}}{c_{65}}} & \frac{c_{16} c_{65}-c_{15} c_{66}}{c_{25} c_{66}-c_{26} c_{65}} & 0 & 0 \\
0 & 0 & 0 & -c_{12} c_{65}-c_{13} c_{66} & c_{65} & c_{66}
\end{array}\right)
$$

and

$$
C_{2}=\left(\begin{array}{cccccc}
-1 & c_{12} & c_{13} & c_{12} c_{15}+c_{13} c_{16} & c_{15} & c_{16} \\
0 & \frac{c_{36}}{c_{36} c_{55}-c_{35} c_{56}} & \frac{c_{35}}{c_{35} c_{56}-c_{36} c_{55}} & \frac{c_{16} c_{35}-c_{15} c_{36}}{c_{35} c_{56}-c_{6} c_{55}} & 0 & 0 \\
0 & 0 & 0 & c_{12} c_{35}+c_{13} c_{36} & c_{35} & c_{36} \\
0 & 0 & 0 & -1 & 0 & 0 \\
0 & 0 & 0 & c_{12} c_{55}+c_{13} c_{56} & c_{55} & c_{56} \\
0 & \frac{c_{56}}{c_{35} c_{56}-c_{36} c_{55}} & \frac{1}{c_{36}-\frac{c_{35} c_{56}}{c_{55}}} & \frac{c_{16} c_{55}-c_{15} c_{56}}{c_{36} c_{55}-c_{35} c_{56}} & 0 & 0
\end{array}\right) .
$$

Although backgrounds calculated from $C_{1}$ and $C_{2}$ seem rather complicated at first sight, dependence on constants $c_{i j}$ can be eliminated in the resulting metrics and $c_{i j}$ appear only in $\widehat{\mathcal{B}}$. Moreover, the torsion vanishes in both cases, hence, up to coordinate or gauge transformations, tensors $\widehat{\mathcal{F}}$ are equivalent to

$$
\widehat{\mathcal{F}}\left(t, \hat{x}_{1}\right)=\left(\begin{array}{cccc}
-1 & 0 & 0 & 0 \\
0 & t^{2} & 0 & 0 \\
0 & 0 & e^{-2 \hat{x}_{1}} t^{2} & 0 \\
0 & 0 & 0 & \frac{e^{2 \hat{x}_{1}}}{t^{2}}
\end{array}\right) .
$$

Such background can be obtained directly if we choose $c_{12}=c_{13}=c_{15}=c_{16}=c_{35}=c_{56}=$ 0 and $c_{36}=c_{55}=1$ in $C_{2}$, in which case we perform "factorized" duality

$$
C_{F}=\left(\begin{array}{cccccc}
-1 & 0 & 0 & 0 & 0 & 0 \\
0 & 1 & 0 & 0 & 0 & 0 \\
0 & 0 & 0 & 0 & 0 & 1 \\
0 & 0 & 0 & -1 & 0 & 0 \\
0 & 0 & 0 & 0 & 1 & 0 \\
0 & 0 & 1 & 0 & 0 & 0
\end{array}\right)
$$

i.e. Buscher duality ${ }^{7}$ in coordinate $y_{3}$.

Tensor (3.13) together with dilaton

$$
\widehat{\Phi}\left(t, \hat{x}_{1}\right)=-\ln t+\hat{x}_{1}
$$

found from (3.2) and $y_{1}=-\hat{x}_{1}$ obtained using (2.22) satisfy standard beta function equations.

\footnotetext{
${ }^{7}$ Duality is accompanied by a change of sign in $\hat{x}_{1}$ that is necessary to get (3.10).
} 
Let us note that metric (3.13) can be brought to the Brinkmann form of a plane-parallel wave

$$
d s^{2}=\frac{2 z_{3}^{2}}{u^{2}} d u^{2}+2 d u d v+d z_{3}^{2}+d z_{4}^{2}
$$

found earlier in [35-37] as non-Abelian T-dual of flat metric.

Backgrounds $\overline{\mathcal{F}}$ on $\overline{\mathscr{G}}$ found from mappings $D_{0} \cdot C_{1}$ and $D_{0} \cdot C_{2}$ can be simplified substantially since after suitable coordinate transformation they differ from background

$$
\overline{\mathcal{F}}\left(t, \bar{x}_{2}, \bar{x}_{3}\right)=\left(\begin{array}{cccc}
-1 & 0 & 0 & 0 \\
0 & \frac{t^{2}}{\left(\bar{x}_{3}^{2}+1\right) t^{4}+\bar{x}_{2}^{2}} & -\frac{\bar{x}_{2}}{\left(\bar{x}_{3}^{2}+1\right) t^{4}+\bar{x}_{2}^{2}} & \frac{\bar{x}_{3} t^{4}}{\left(\bar{x}_{3}^{2}+1\right) t^{4}+\bar{x}_{2}^{2}} \\
0 & \frac{\bar{x}_{2}}{\left(\bar{x}_{3}^{2}+1\right) t^{4}+\bar{x}_{2}^{2}} & \frac{\left(\bar{x}_{3}^{2}+1\right) t^{2}}{\left(\bar{x}_{3}^{2}+1\right) t^{4}+\bar{x}_{2}^{2}} & \frac{\bar{x}_{2} \bar{x}_{3} t^{2}}{\left(\bar{x}_{3}^{2}+1\right) t^{4}+\bar{x}_{2}^{2}} \\
0 & -\frac{\bar{x}_{3} t^{4}}{\left(\bar{x}_{3}^{2}+1\right) t^{4}+\bar{x}_{2}^{2}} & \frac{\bar{x}_{2} \bar{x}_{3} t^{2}}{\left(\bar{x}_{3}^{2}+1\right) t^{4}+\bar{x}_{2}^{2}} & \frac{t^{2}\left(t^{4}+\bar{x}_{2}^{2}\right)}{\left(\bar{x}_{3}^{2}+1\right) t^{4}+\bar{x}_{2}^{2}}
\end{array}\right)
$$

obtained using $D_{0} \cdot C_{F}$ only by a constant shift of the $\overline{\mathcal{B}}$ field. Since $y_{1}=-\hat{x}_{1}$, we find dilaton and vector $\overline{\mathcal{J}}$ as

$$
\bar{\Phi}\left(t, \bar{x}_{2}, \bar{x}_{3}\right)=-\frac{1}{2} \ln \left(t^{4}\left(\bar{x}_{3}^{2}+1\right)+\bar{x}_{2}^{2}\right), \quad \overline{\mathcal{J}}=(0,-1,0,0)
$$

and the Generalized Supergravity Equations (2.17)-(2.19) are satisfied. On the other hand, we have $\operatorname{Div} \Theta=(0,0,0,0) \neq \overline{\mathcal{J}}$.

\subsubsection{Transformation of $\mathfrak{b}_{V} \bowtie \mathfrak{a}$ to $\mathfrak{b}_{V} \bowtie \mathfrak{b}_{I I}$ and to its dual}

Lie algebra $\mathfrak{d}=\mathfrak{b}_{V} \bowtie \mathfrak{b}_{I I}$ is spanned by $\left(\widehat{T}_{1}, \widehat{T}_{2}, \widehat{T}_{3}, \bar{T}^{1}, \bar{T}^{2}, \bar{T}^{3}\right)$ with algebraic relations

$$
\begin{aligned}
& {\left[\widehat{T}_{1}, \widehat{T}_{2}\right]=\widehat{T}_{2}, \quad\left[\widehat{T}_{1}, \widehat{T}_{3}\right]=\widehat{T}_{3}, \quad\left[\widehat{T}_{1}, \bar{T}^{2}\right]=\widehat{T}_{3}-\bar{T}^{2},} \\
& {\left[\widehat{T}_{1}, \bar{T}^{3}\right]=-\widehat{T}_{2}-\bar{T}^{3}, \quad\left[\widehat{T}_{2}, \bar{T}^{2}\right]=\bar{T}^{1}, \quad\left[\widehat{T}_{3}, \bar{T}^{3}\right]=\bar{T}^{1}, \quad\left[\bar{T}^{2}, \bar{T}^{3}\right]=\bar{T}^{1} .}
\end{aligned}
$$

Left-invariant vector fields of $\widehat{\mathscr{G}}$ and $\overline{\mathscr{G}}$ are

$$
\begin{array}{lll}
\widehat{V}_{1}=\partial_{\hat{x}_{1}}-\hat{x}_{2} \partial_{\hat{x}_{2}}-\hat{x}_{3} \partial_{\hat{x}_{3},} & \widehat{V}_{2}=\partial_{\hat{x}_{2},}, \widehat{V}_{3}=\partial_{\hat{x}_{3}}, \\
\bar{V}_{1}=\partial_{\bar{x}_{1}}, & \bar{V}_{2}=-\bar{x}_{3} \partial_{\bar{x}_{1}}+\partial_{\bar{x}_{2}}, & \bar{V}_{3}=\partial_{\bar{x}_{3}} .
\end{array}
$$

Mappings $C$ that transform the algebraic relations of Manin triple $\mathfrak{b}_{V} \bowtie \mathfrak{a}$ to $\mathfrak{b}_{V} \bowtie \mathfrak{b}_{I I}$ are given by matrices

$$
C_{1}=\left(\begin{array}{cccccc}
1 & c_{12} & c_{13} & -c_{12} c_{15}-c_{13} c_{16} & c_{15} & c_{16} \\
0 & c_{22} & c_{23} & -c_{15} c_{22}-c_{16} c_{23} & 0 & 0 \\
0 & c_{32} & c_{33} & -c_{15} c_{32}-c_{16} c_{33} & 0 & 0 \\
0 & 0 & 0 & 1 & 0 & 0 \\
0 & \frac{c_{32}}{2} & \frac{c_{33}}{2} & \Gamma_{54} & \frac{c_{33}}{c_{22} c_{33}-c_{23} c_{32}} & \frac{c_{32}}{c_{23} c_{32}-c_{22} c_{33}} \\
0 & -\frac{c_{22}}{2} & -\frac{c_{23}}{2} & \Gamma_{64} & \frac{c_{23}}{c_{23} c_{32}-c_{22} c_{33}} & \frac{c_{22} c_{33}-c_{23} c_{32}}{c_{2}}
\end{array}\right)
$$

where

$$
\begin{aligned}
& \Gamma_{54}=\frac{2 c_{13} c_{32}+c_{15}\left(c_{23} c_{32}-c_{22} c_{33}\right) c_{32}+c_{33}\left(c_{16}\left(c_{23} c_{32}-c_{22} c_{33}\right)-2 c_{12}\right)}{2 c_{22} c_{33}-2 c_{23} c_{32}}, \\
& \Gamma_{64}=\frac{-2 c_{13} c_{22}+2 c_{12} c_{23}+\left(c_{15} c_{22}+c_{16} c_{23}\right)\left(c_{22} c_{33}-c_{23} c_{32}\right)}{2 c_{22} c_{33}-2 c_{23} c_{32}}
\end{aligned}
$$


and

where

$$
C_{2}=\left(\begin{array}{cccccc}
-1 & c_{12} & c_{13} & c_{12} c_{15}+c_{13} c_{16} & c_{15} & c_{16} \\
0 & 0 & 0 & c_{12} c_{25}+c_{13} c_{26} & c_{25} & c_{26} \\
0 & 0 & 0 & c_{12} c_{35}+c_{13} c_{36} & c_{35} & c_{36} \\
0 & 0 & 0 & -1 & 0 & 0 \\
0 & \frac{c_{36}}{c_{25} c_{36}-c_{26} c_{35}} & \frac{1}{c_{26}-\frac{c_{25} c_{36}}{c_{35}}} & \gamma_{54} & \frac{c_{35}}{2} & \frac{c_{36}}{2} \\
0 & \frac{c_{26}}{c_{26} c_{35}-c_{25} c_{36}} & \frac{c_{25}}{c_{25} c_{36}-c_{26} c_{35}} & \gamma_{64} & -\frac{c_{25}}{2} & -\frac{c_{26}}{2}
\end{array}\right)
$$

$$
\begin{aligned}
\gamma_{54} & =\frac{-2 c_{16} c_{35}+c_{12}\left(c_{25} c_{36}-c_{26} c_{35}\right) c_{35}+c_{36}\left(2 c_{15}+c_{13}\left(c_{25} c_{36}-c_{26} c_{35}\right)\right)}{2 c_{25} c_{36}-2 c_{26} c_{35}}, \\
\gamma_{64} & =\frac{-2 c_{16} c_{25}+2 c_{15} c_{26}+\left(c_{12} c_{25}+c_{13} c_{26}\right)\left(c_{25} c_{36}-c_{26} c_{35}\right)}{2 c_{26} c_{35}-2 c_{25} c_{36}}
\end{aligned}
$$

For $c_{12}=c_{13}=c_{15}=c_{16}=c_{23}=c_{32}=0$ and $c_{22}=c_{33}=1$ the matrix $C_{1}$ is equal to $\beta$-shift

$$
C_{10}=\left(\begin{array}{cccccc}
1 & 0 & 0 & 0 & 0 & 0 \\
0 & 1 & 0 & 0 & 0 & 0 \\
0 & 0 & 1 & 0 & 0 & 0 \\
0 & 0 & 0 & 1 & 0 & 0 \\
0 & 0 & \frac{1}{2} & 0 & 1 & 0 \\
0 & -\frac{1}{2} & 0 & 0 & 0 & 1
\end{array}\right)
$$

Background

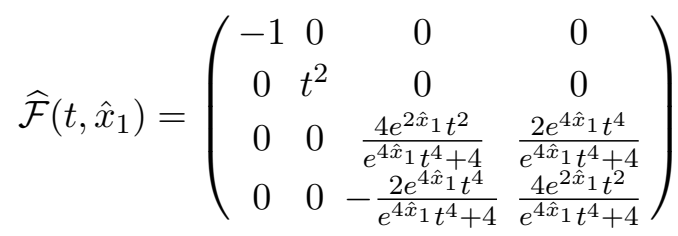

obtained by this $\beta$-shift can be brought to the Brinkmann form of a plane parallel wave

$$
d s^{2}=\frac{2 u^{2}\left(u^{4}-5\right)\left(z_{3}^{2}+z_{4}^{2}\right)}{\left(u^{4}+1\right)^{2}} d u^{2}+2 d u d v+d z_{3}^{2}+d z_{4}^{2}
$$

accompanied by a non-trivial torsion

$$
\widehat{H}=\frac{4 u}{1+u^{4}} d u \wedge d z_{3} \wedge d z_{4} .
$$

This background was found in [37] as non-Abelian T-dual of flat metric. Dilaton that together with (3.16) satisfies beta function equations is

$$
\hat{\Phi}\left(t, \hat{x}_{1}\right)=-\frac{1}{2} \ln \left(\frac{t^{4} e^{4 \hat{x}_{1}}}{4}+1\right) .
$$

Dual of (3.16) calculated using $D_{0} \cdot C_{10}$ reads

$$
\overline{\mathcal{F}}\left(t, \bar{x}_{2}, \bar{x}_{3}\right)=\left(\begin{array}{cccc}
-1 & 0 & 0 & 0 \\
0 & \frac{t^{2}}{t^{4}+\bar{x}_{2}^{2}+\bar{x}_{3}^{2}} & \frac{\bar{x}_{3} t^{2}+2 \bar{x}_{2}}{2\left(t^{4}+\bar{x}_{2}^{2}+\bar{x}_{3}^{2}\right)} & \frac{\bar{x}_{2} t^{2}+2 \bar{x}_{3}}{2\left(t^{4}+\bar{x}_{2}^{2}+\bar{x}_{3}^{2}\right)} \\
0 & \frac{t^{2} \bar{x}_{3}-2 \bar{x}_{2}}{2\left(t^{4}+\bar{x}_{2}^{2}+\bar{x}_{3}^{2}\right)} & \frac{\left(\bar{x}_{3}^{2}+4\right) t^{4}+4 \bar{x}_{3}^{2}}{4 t^{2}\left(t^{4}+\bar{x}_{2}^{2}+\bar{x}_{3}^{2}\right)} & \frac{-2 t^{6}+\bar{x}_{2} \bar{x}_{3} t^{4}-4 \bar{x}_{2}^{2} t^{2}-4 \bar{x}_{2} \bar{x}_{3}}{4 t^{2}\left(t^{4}+\bar{x}_{2}^{2}+\bar{x}_{3}^{2}\right)} \\
0 & \frac{t^{2} \bar{x}_{2}-2 \bar{x}_{3}}{2\left(t^{4}+\bar{x}_{2}^{2}+\bar{x}_{3}^{2}\right)} & \frac{2 t^{6}+\bar{x}_{2} \bar{x}_{3} t^{4}+4 \bar{x}_{2}^{2} t^{2}-4 \bar{x}_{2} \bar{x}_{3}}{4 t^{2}\left(t^{4}+\bar{x}_{2}^{2}+\bar{x}_{3}^{2}\right)} & \frac{\left(\bar{x}_{2}^{2}+4\right) t^{4}+4 \bar{x}_{2}^{2}}{4 t^{2}\left(t^{4}+\bar{x}_{2}^{2}+\bar{x}_{3}^{2}\right)}
\end{array}\right),
$$


and with dilaton

$$
\bar{\Phi}\left(t, \bar{x}_{2}, \bar{x}_{3}\right)=-\frac{1}{2} \ln \left(t^{2}\left(t^{4}+\bar{x}_{2}^{2}+\bar{x}_{3}^{2}\right)\right)
$$

it satisfies Generalized Supergravity Equations with $\overline{\mathcal{J}}=(0,2,0,0)$. However, Div $\Theta=$ $\left(0, \frac{8}{4+t^{4}}, 0,0\right)$.

For $c_{12}=c_{13}=c_{15}=c_{16}=c_{26}=c_{35}=0$ and $c_{25}=c_{36}=1$ the matrix $C_{2}$ is equal to $\beta$-shift followed by factorized duality

$$
C_{20}=\left(\begin{array}{cccccc}
-1 & 0 & 0 & 0 & 0 & 0 \\
0 & 0 & 0 & 0 & 1 & 0 \\
0 & 0 & 0 & 0 & 0 & 1 \\
0 & 0 & 0 & -1 & 0 & 0 \\
0 & 1 & 0 & 0 & 0 & \frac{1}{2} \\
0 & 0 & 1 & 0 & -\frac{1}{2} & 0
\end{array}\right)
$$

Background obtained by transformation (3.20) is given by tensor

$$
\widehat{\mathcal{F}}\left(t, \hat{x}_{1}\right)=\left(\begin{array}{cccc}
-1 & 0 & 0 & 0 \\
0 & t^{2} & 0 & 0 \\
0 & 0 & \frac{4 e^{2 \hat{x}_{1}} t^{2}}{4 t^{4}+e^{4 \hat{x}_{1}}} & \frac{2 e^{4 \hat{x}_{1}}}{4 t^{4}+e^{4 \hat{x}_{1}}} \\
0 & 0 & -\frac{2 e^{4 \hat{x}_{1}}}{4 t^{4}+e^{4 \hat{x}_{1}}} & \frac{4 e^{2} x_{1}}{4 t^{4}+e^{4} \hat{x}_{1}}
\end{array}\right)
$$

that again gives plane parallel wave (3.17) with torsion (3.18). Dilaton that together with (3.21) satisfy beta function equations is

$$
\widehat{\Phi}\left(t, \hat{x}_{1}\right)=-\frac{1}{2} \ln \left(t^{4} e^{-4 \hat{x}_{1}}+\frac{1}{4}\right) .
$$

Dual of (3.21) found from $D_{0} \cdot C_{20}$ is

$$
\overline{\mathcal{F}}\left(t, \bar{x}_{2}, \bar{x}_{3}\right)=\left(\begin{array}{cccc}
-1 & 0 & 0 & 0 \\
0 & \frac{1}{t^{2}\left(\bar{x}_{2}^{2}+\bar{x}_{3}^{2}+1\right)} & \frac{2 \bar{x}_{2} t^{2}+\bar{x}_{3}}{2 t^{2}\left(\bar{x}_{2}^{2}+\bar{x}_{3}^{2}+1\right)} & \frac{2 \bar{x}_{3} t^{2}+\bar{x}_{2}}{2 t^{2}\left(\bar{x}_{2}^{2}+\bar{x}_{3}^{2}+1\right)} \\
0 & \frac{\bar{x}_{3}-2 t^{2} \bar{x}_{2}}{2 t^{2}\left(\bar{x}_{2}^{2}+\bar{x}_{3}^{2}+1\right)} & \frac{4\left(\bar{x}_{3}^{2}+1\right) t^{4}+\bar{x}_{3}^{2}}{4 t^{2}\left(\bar{x}_{2}^{2}+\bar{x}_{3}^{2}+1\right)} & \frac{-4 \bar{x}_{2} x_{3} t^{4}-2\left(2 \bar{x}_{2}^{2}+1\right) t^{2}+\bar{x}_{2} \bar{x}_{3}}{4 t^{2}\left(\bar{x}_{2}^{2}+\bar{x}_{3}^{2}+1\right)} \\
0 & \frac{\bar{x}_{2}-2 t^{2} \bar{x}_{3}}{2 t^{2}\left(\bar{x}_{2}^{2}+\bar{x}_{3}^{2}+1\right)} & \frac{-4 \bar{x}_{2} \bar{x}_{3} t^{4}+\left(4 \bar{x}_{2}^{2}+2\right) t^{2}+\bar{x}_{2} \bar{x}_{3}}{4 t^{2}\left(\bar{x}_{2}^{2}+\bar{x}_{3}^{2}+1\right)} & \frac{4\left(\bar{x}_{2}^{2}+1\right) t^{4}+\bar{x}_{2}^{2}}{4 t^{2}\left(\bar{x}_{2}^{2}+\bar{x}_{3}^{2}+1\right)}
\end{array}\right) .
$$

It is torsionless and with dilaton

$$
\bar{\Phi}\left(t, \bar{x}_{2}, \bar{x}_{3}\right)=-\frac{1}{2} \ln \left(t^{2}\left(\bar{x}_{2}^{2}+\bar{x}_{3}^{2}+1\right)\right)
$$

it satisfies beta function equations, i.e. Generalized Supergravity Equations with $\overline{\mathcal{J}}=$ $(0,0,0,0)$. However, Div $\Theta=\left(0, \frac{8 t^{4}}{1+4 t^{4}}, 0,0\right)$. 


\subsubsection{Transformation of $\mathfrak{b}_{V} \bowtie \mathfrak{a}$ to $\mathfrak{b}_{V I_{-1}} \bowtie \mathfrak{b}_{V i i}$ and to its dual}

Lie algebra $\mathfrak{d}=\mathfrak{b}_{V I_{-1}} \bowtie \mathfrak{b}_{V i i}$ is spanned by basis $\left(\widehat{T}_{1}, \widehat{T}_{2}, \widehat{T}_{3}, \bar{T}^{1}, \bar{T}^{2}, \bar{T}^{3}\right)$ with algebraic relations

$$
\begin{aligned}
& {\left[\widehat{T}_{1}, \widehat{T}_{2}\right]=-\widehat{T}_{2}, \quad\left[\widehat{T}_{1}, \widehat{T}_{3}\right]=\widehat{T}_{3},} \\
& {\left[\widehat{T}_{1}, \bar{T}^{1}\right]=-\widehat{T}_{2}, \quad\left[\widehat{T}_{1}, \bar{T}^{2}\right]=\widehat{T}_{1}+\bar{T}^{2}, \quad\left[\widehat{T}_{1}, \bar{T}^{3}\right]=-\bar{T}^{3},} \\
& {\left[\widehat{T}_{2}, \bar{T}^{2}\right]=-\bar{T}^{1}, \quad\left[\widehat{T}_{3}, \bar{T}^{2}\right]=\widehat{T}_{3}, \quad\left[\widehat{T}_{3}, \bar{T}^{3}\right]=-\widehat{T}_{2}+\bar{T}^{1},} \\
& {\left[\bar{T}^{1}, \bar{T}^{2}\right]=-\bar{T}^{1}, \quad\left[\bar{T}^{2}, \bar{T}^{3}\right]=\bar{T}^{3} .}
\end{aligned}
$$

Left-invariant vector fields of $\widehat{\mathscr{G}}$ and $\overline{\mathscr{G}}$ are

$$
\begin{array}{lll}
\widehat{V}_{1}=\partial_{\hat{x}_{1}}+\hat{x}_{2} \partial_{\hat{x}_{2}}-\hat{x}_{3} \partial_{\hat{x}_{3},} & \widehat{V}_{2}=\partial_{\hat{x}_{2},} & \widehat{V}_{3}=\partial_{\hat{x}_{3}}, \\
\bar{V}_{1}=e^{\bar{x}_{2}} \partial_{\bar{x}_{1}}, & \bar{V}_{2}=\partial_{\bar{x}_{2}}-\bar{x}_{3} \partial_{\bar{x}_{3}}, & \bar{V}_{3}=\partial_{\bar{x}_{3}} .
\end{array}
$$

Examples of mappings $C$ that transform the algebraic structure of Manin triple $\mathfrak{b}_{V I_{-1}} \bowtie \mathfrak{a}$ to $\mathfrak{b}_{V I_{-1}} \bowtie \mathfrak{b}_{V i i}$ are given by matrices

$$
\begin{gathered}
C_{1}=\left(\begin{array}{cccccc}
-1 & 0 & 0 & 0 & 0 & 0 \\
0 & 1 & 0 & 0 & 0 & 0 \\
0 & 0 & 0 & 0 & 0 & 1 \\
0 & 1 & 0 & -1 & 0 & 0 \\
1 & 0 & 0 & 0 & 1 & 0 \\
0 & 0 & 1 & 0 & 0 & 0
\end{array}\right), \\
C_{2}=\left(\begin{array}{cccccc}
1 & 0 & 0 & 0 & 0 & 0 \\
0 & 0 & 0 & 0 & 1 & 0 \\
0 & 0 & 1 & 0 & 0 & 0 \\
0 & 0 & 0 & 1 & 1 & 0 \\
-1 & 1 & 0 & 0 & 0 & 0 \\
0 & 0 & 0 & 0 & 0 & 1
\end{array}\right)
\end{gathered}
$$

Background obtained by $C_{1}$ is given by tensor

$$
\widehat{\mathcal{F}}\left(t, \hat{x}_{1}, \hat{x}_{3}\right)=\left(\begin{array}{cccc}
-1 & 0 & 0 & 0 \\
0 & \frac{e^{2 \hat{x}_{1}}\left(\hat{x}_{3}^{2}+1\right) t^{2}}{t^{4}+e^{2 \hat{x}_{1}\left(\hat{x}_{3}^{2}+1\right)}} & \frac{t^{4}}{t^{4}+e^{2 \hat{x}_{1}}\left(\hat{x}_{3}^{2}+1\right)} & \frac{e^{21_{1} \hat{x}_{3} t^{2}}}{t^{4}+e^{2 \hat{x}_{1}}\left(\hat{x}_{3}^{2}+1\right)} \\
0 & -\frac{t^{4}}{t^{4}+e^{2 \hat{x}_{1}}\left(\hat{x}_{3}^{2}+1\right)} & \frac{t^{2}}{t^{4}+e^{2 \hat{x}_{1}}\left(\hat{x}_{3}^{2}+1\right)} & \frac{e^{2 \hat{x}_{1}} \hat{x}_{3}}{t^{4}+e^{2 \hat{x}_{1}}\left(\hat{x}_{3}^{2}+1\right)} \\
0 & \frac{e^{2 \hat{x}_{1}} \hat{x}_{3} t^{2}}{t^{4}+e^{2 \hat{x}} 1\left(\hat{x}_{3}^{2}+1\right)} & -\frac{e^{2 \hat{x}_{1}} \hat{x}_{3}}{t^{4}+e^{2 \hat{x}} 1\left(\hat{x}_{3}^{2}+1\right)} & \frac{e^{2 \hat{x}_{1}}\left(t^{4}+e^{2 \hat{x}_{1}}\right)}{t^{2}\left(t^{4}+e^{2 \hat{x}_{1}}\left(\hat{x}_{3}^{2}+1\right)\right)}
\end{array}\right) .
$$

Together with the dilaton

$$
\widehat{\Phi}\left(t, \hat{x}_{1}, \hat{x}_{3}\right)=-\frac{1}{2} \ln \left(t^{6} e^{-4 \hat{x}_{1}}+t^{2} e^{-2 \hat{x}_{1}}\left(\hat{x}_{3}^{2}+1\right)\right)
$$

they satisfy Generalized Supergravity Equations with $\widehat{\mathcal{J}}=(0,0,2,0)$, but $\operatorname{Div} \Theta=$ $(0,0,1,0)$. 
Dual of (3.24)

$$
\begin{aligned}
& \overline{\mathcal{F}}\left(t, \bar{x}_{1}, \bar{x}_{2}, \bar{x}_{3}\right)= \\
& =\left(\begin{array}{cccc}
-1 & 0 & 0 & 0 \\
0 & \frac{t^{2}}{\Delta} & -\frac{\bar{x}_{1} t^{2}+e^{\bar{x}_{2}}\left(t^{4}+1\right)-1}{\Delta} & \frac{e^{3 \bar{x}_{2} \bar{x}_{3} t^{4}}}{\Delta} \\
0 & \frac{-\bar{x}_{1} t^{2}+e^{x_{2}}\left(t^{4}+1\right)-1}{\Delta} & \frac{t^{2}\left(\bar{x}_{1}^{2}+e^{4 \bar{x}_{2}} \bar{x}_{3}^{2}\left(t^{4}+1\right)+1\right)}{\Delta} & \frac{e^{3 \bar{x}_{2}} \bar{x}_{3} t^{2}\left(-\bar{x}_{1} t^{2}+e^{\bar{x}_{2}}\left(t^{4}+1\right)-1\right)}{\Delta} \\
0 & -\frac{e^{3 \bar{x}_{2}} \bar{x}_{3} t^{4}}{\Delta} & \frac{e^{3 \bar{x}_{2}} \bar{x}_{3} t^{2}\left(\bar{x}_{1} t^{2}+\bar{x}^{\bar{x}_{2}}\left(t^{4}+1\right)-1\right)}{\Delta} & \frac{e^{2 \bar{x}_{2}} t^{2}\left(e^{2 \bar{x}_{2}}\left(t^{4}+1\right)-2 e^{\bar{x}_{2}}+1\right)}{\Delta}
\end{array}\right)
\end{aligned}
$$

where

$$
\Delta=e^{4 \bar{x}_{2}} \bar{x}_{3}^{2} t^{4}-2 e^{\bar{x}_{2}}+e^{2 \bar{x}_{2}}\left(t^{4}+1\right)+1
$$

and dilaton

$$
\bar{\Phi}\left(t, \bar{x}_{2}, \bar{x}_{3}\right)=-\frac{1}{2} \ln \Delta
$$

calculated by the formula (2.20) satisfy Generalized Supergravity Equations with vector $\overline{\mathcal{J}}=\left(0,-e^{\bar{x}_{2}}, 0,0\right)$ but $\operatorname{Div} \Theta=\left(0, \frac{1}{t^{4}+1}-e^{\bar{x}_{2}}, 0,0\right)$.

Background obtained by $C_{2}$ is torsionless and has the form

$$
\widehat{\mathcal{F}}\left(t, \hat{x}_{1}, \hat{x}_{3}\right)=\left(\begin{array}{cccc}
-1 & 0 & 0 & 0 \\
0 & \frac{e^{2 \hat{x}_{1}}\left(\hat{x}_{3}^{2}+1\right) t^{2}}{e^{2 \hat{x}_{1}}\left(\hat{x}_{3}^{2}+1\right)+1} & \frac{1}{e^{2 \hat{x}_{1}}\left(\hat{x}_{3}^{2}+1\right)+1} & \frac{e^{2 \hat{x}_{1}} \hat{x}_{3} t^{2}}{e^{2 \hat{x}_{1}}\left(\hat{x}_{3}^{2}+1\right)+1} \\
0 & -\frac{1}{e^{2 \hat{x}_{1}}\left(\hat{x}_{3}^{2}+1\right)+1} & \frac{1}{\left.e^{2 \hat{x}_{1}}\left(\hat{x}_{3}^{2}+1\right)+1\right) t^{2}} & \frac{e^{2 x_{1}} \hat{x}_{3}}{e^{2 \hat{x}_{1}}\left(\hat{x}_{3}^{2}+1\right)+1} \\
0 & \frac{e^{2 \hat{x}_{1}} \hat{x}_{3} t^{2}}{e^{2 \hat{x}_{1}\left(\hat{x}_{3}^{2}+1\right)+1}} & -\frac{e^{2 \hat{x}_{1}} \hat{x}_{3}}{e^{2 \hat{x}_{1}}\left(\hat{x}_{3}^{2}+1\right)+1} & \frac{e^{2 \hat{x}_{1}}\left(1+e^{2 \hat{x}_{1}}\right) t^{2}}{e^{2 \hat{x}_{1}}\left(\hat{x}_{3}^{2}+1\right)+1}
\end{array}\right) .
$$

Together with dilaton

$$
\widehat{\Phi}\left(t, \hat{x}_{1}, \hat{x}_{3}\right)=-\frac{1}{2} \ln \left(t^{2}\left(1+e^{2 \hat{x}_{1}}\left(\hat{x}_{3}^{2}+1\right)\right)\right)
$$

it satisfies Generalized Supergravity Equations with vector $\widehat{\mathcal{J}}=(0,0,0,0)$, but $\operatorname{Div} \Theta=$ $(0,0,1,0)$.

Dual of (3.27) given by

$$
\overline{\mathcal{F}}\left(t, \bar{x}_{1}, \bar{x}_{2}, \bar{x}_{3}\right)=\left(\begin{array}{cccc}
-1 & 0 & 0 & 0 \\
0 & \frac{t^{2}}{\Delta} & \frac{\left(1-2 e^{\bar{x}_{2}}\right) t^{4}-t^{2} \bar{x}_{1}}{\Delta} & \frac{e^{3 \bar{x}_{2} \bar{x}_{3}}}{\Delta} \\
0 & \frac{\left(-1+2 e^{\bar{x}_{2}}\right) t^{4}-t^{2} \bar{x}_{1}}{\Delta} & \frac{t^{2}\left(t^{4}+\bar{x}_{1}^{2}+2 e^{4 \bar{x}_{2}} \bar{x}_{3}^{2}\right)}{\Delta} & \frac{e^{3 \bar{x}_{2}}\left(\left(-1+2 e^{\bar{x}_{2}}\right) t^{2}-\bar{x}_{1}\right) \bar{x}_{3}}{\Delta} \\
0 & -\frac{e^{3 \bar{x}_{2} \bar{x}_{3}}}{\Delta} & \frac{e^{3 \bar{x}_{2}}\left(\left(-1+2 e^{\bar{x}_{2}}\right) t^{2}+\bar{x}_{1}\right) \bar{x}_{3}}{\Delta} & \frac{e^{2 \bar{x}_{2}}\left(1-2 e^{\frac{\Delta}{x_{2}}}+2 e^{2 \bar{x}_{2}}\right) t^{2}}{\Delta}
\end{array}\right)
$$

where

$$
\Delta=\left(1-2 e^{\bar{x}_{2}}+2 e^{2 \bar{x}_{2}}\right) t^{4}+e^{4 \bar{x}_{2}} \bar{x}_{3}^{2}
$$

and dilaton

$$
\bar{\Phi}\left(t, \bar{x}_{2}, \bar{x}_{3}\right)=-\frac{1}{2} \ln \left(t^{4}\left(e^{-4 \bar{x}_{2}}-2 e^{-3 \bar{x}_{2}}+2 e^{-2 \bar{x}_{2}}\right)+\bar{x}_{3}^{2}\right)
$$

calculated by formula (2.20) satisfy Generalized Supergravity Equations with vector $\overline{\mathcal{J}}=$ $\left(0, e^{\bar{x}_{2}}, 0,0\right)$. On the other hand, Div $\Theta=\left(0, \frac{1}{2}-e^{\bar{x}_{2}}, 0,0\right)$. 


\section{Poisson-Lie T-plurality of curved cosmologies}

\subsection{Bianchi $V I_{-1}$ cosmology}

Next we will transform curved Bianchi $V I_{-1}$ cosmology given by metric

$$
\mathcal{F}\left(t, y_{1}\right)=\left(\begin{array}{cccc}
-e^{-4 \Phi(t)} a_{1}(t)^{2} a_{2}(t)^{4} & 0 & 0 & 0 \\
0 & a_{1}(t)^{2} & 0 & 0 \\
0 & 0 & e^{-2 y_{1}} a_{2}(t)^{2} & 0 \\
0 & 0 & 0 & e^{2 y_{1}} a_{2}(t)^{2}
\end{array}\right)
$$

where

$$
a_{1}(t)=\sqrt{p_{1}} \exp \left(\frac{1}{2} e^{2 p_{2} t}+\frac{p_{1} t}{2}+\Phi(t)\right), \quad a_{2}(t)=\sqrt{p_{2}} e^{\frac{p_{2} t}{2}+\Phi(t)}
$$

and dilaton $\Phi=\beta t$. Its scalar curvature is

$$
R=\frac{\left(-12 \beta^{2}+2 p_{1} p_{2}+p_{2}^{2}\right) e^{-t\left(p_{1}+2\left(\beta+p_{2}\right)\right)-e^{2 p_{2} t}}}{2 p_{1} p_{2}^{2}}
$$

and beta function equations reduce to condition

$$
\beta^{2}=\frac{1}{4}\left(2 p_{1} p_{2}+p_{2}^{2}\right)
$$

for constants $p_{1}, p_{2}$ and $\beta$.

The metric is invariant with respect to the action of Bianchi $V I_{-1}$ group and can be constructed by virtue of Manin triple $\mathfrak{d}=\mathfrak{b}_{V I_{-1}} \bowtie \mathfrak{a}$ spanned by basis $\left(T_{1}, T_{2}, T_{3}, \widetilde{T}^{1}, \widetilde{T}^{2}, \widetilde{T}^{3}\right)$ with algebraic relations

$$
\begin{aligned}
& {\left[T_{1}, T_{2}\right]=-T_{2},} \\
& {\left[T_{1}, T_{3}\right]=T_{3},} \\
& {\left[T_{1}, \widetilde{T}^{2}\right]=\widetilde{T}^{2},} \\
& {\left[T_{1}, \widetilde{T}^{3}\right]=-\widetilde{T}^{3},} \\
& {\left[T_{2}, \widetilde{T}^{2}\right]=-\widetilde{T}^{1},} \\
& {\left[T_{3}, \widetilde{T}^{3}\right]=\widetilde{T}^{1} \text {. }}
\end{aligned}
$$

Structure constants of $\mathfrak{b}_{V I_{-1}}$ are traceless.

Drinfel'd double is the same as in section 3.2, where we have seen that beside $\mathfrak{d}=$ $\mathfrak{b}_{V I_{-1}} \bowtie \mathfrak{a}$ the algebra $\mathfrak{d}$ can be decomposed into Manin triples $\mathfrak{b}_{V} \bowtie \mathfrak{a}, \mathfrak{b}_{V} \bowtie \mathfrak{b}_{I I}, \mathfrak{b}_{V I_{-1}} \bowtie$ $\mathfrak{b}_{V . i i}$ and their duals. In this section, however, the backgrounds are different as we use different matrix $E(s)$.

Formula (2.20) for new dilatons

$$
\widehat{\Phi}(t, \hat{x})=\beta t-\frac{1}{2} \ln |\operatorname{det}[(N+\widehat{\Pi}(\hat{x}) M) \widehat{a}(\hat{x})]|
$$

does not depend on coordinates $y$ and is, therefore, applicable for any Manin triple of this Drinfel'd double. 


\subsubsection{Transformation of $\mathfrak{b}_{V I_{-1}} \bowtie \mathfrak{a}$ to $\mathfrak{b}_{V} \bowtie \mathfrak{a}$ and to its dual}

Lie algebra $\mathfrak{d}=\mathfrak{b}_{V} \bowtie \mathfrak{a}$ is spanned by $\left(\widehat{T}_{1}, \widehat{T}_{2}, \widehat{T}_{3}, \bar{T}^{1}, \bar{T}^{2}, \bar{T}^{3}\right)$ with the algebraic relations

$$
\begin{array}{rlrl}
{\left[\widehat{T}_{1}, \widehat{T}_{2}\right]} & =\widehat{T}_{2}, & {\left[\widehat{T}_{1}, \widehat{T}_{3}\right]=\widehat{T}_{3},} & {\left[\widehat{T}_{1}, \bar{T}^{2}\right]=-\bar{T}^{2},} \\
{\left[\widehat{T}_{1}, \bar{T}^{3}\right]=-\bar{T}^{3},} & {\left[\widehat{T}_{2}, \bar{T}^{2}\right]=\bar{T}^{1},} & {\left[\widehat{T}_{3}, \bar{T}^{3}\right]=\bar{T}^{1} .}
\end{array}
$$

Left-invariant vector fields of $\widehat{\mathscr{G}}$ and $\overline{\mathscr{G}}$ are

$$
\begin{array}{lll}
\widehat{V}_{1}=\partial_{\hat{x}_{1}}-\hat{x}_{2} \partial_{\hat{x}_{2}}-\hat{x}_{3} \partial_{\hat{x}_{3}}, & \widehat{V}_{2}=\partial_{\hat{x}_{2}}, & \widehat{V}_{3}=\partial_{\hat{x}_{3}}, \\
\bar{V}_{1}=\partial_{\bar{x}_{1}}, & \bar{V}_{2}=\partial_{\bar{x}_{2}}, & \bar{V}_{3}=\partial_{\bar{x}_{3}} .
\end{array}
$$

Mappings $C$ that transform the algebraic structure of Manin triple $\mathfrak{b}_{V I_{-1}} \bowtie \mathfrak{a}$ to $\mathfrak{b}_{V} \bowtie \mathfrak{a}$ are given by matrices inverse to (3.11) and (3.12). After a suitable change of coordinates we find that backgrounds obtained from these general solutions differ from background

$$
\widehat{\mathcal{F}}\left(t, \hat{x}_{1}\right)=\left(\begin{array}{cccc}
-e^{-4 t \beta} a_{1}(t)^{2} a_{2}(t)^{4} & 0 & 0 & 0 \\
0 & a_{1}(t)^{2} & 0 & 0 \\
0 & 0 & e^{2 \hat{x}_{1}} a_{2}(t)^{2} & 0 \\
0 & 0 & 0 & \frac{e^{2 \hat{x}_{1}}}{a_{2}(t)^{2}}
\end{array}\right)
$$

obtained using factorized duality (3.14) only by a torsionless $\widehat{\mathcal{B}}$ field. Note that $\widehat{\mathcal{F}}$ is again invariant with respect to group $\mathscr{B}_{V}$. This metric is not flat and dilaton that together with (4.3) satisfy beta function equations is

$$
\widehat{\Phi}\left(t, \hat{x}_{1}\right)=\beta t+\hat{x}_{1}-\ln a_{2}(t) .
$$

Dual of (4.3) is given by tensor

$$
\overline{\mathcal{F}}\left(t, \bar{x}_{2}, \bar{x}_{3}\right)=\left(\begin{array}{cccc}
-e^{-4 \beta t} a_{1}(t)^{2} a_{2}(t)^{4} & 0 & 0 & 0 \\
0 & \frac{a_{2}(t)^{2}}{\Delta} & \frac{\bar{x}_{2}}{\Delta} & \frac{a_{2}(t)^{4} \bar{x}_{3}}{\Delta} \\
0 & -\frac{\bar{x}_{2}}{\Delta} & \frac{a_{1}(t)^{2}+a_{2}(t)^{2} \bar{x}_{3}^{2}}{\Delta} & -\frac{a_{2}(t)^{2} \bar{x}_{2} \bar{x}_{3}}{\Delta} \\
0 & -\frac{a_{2}(t)^{4} \bar{x}_{3}}{\Delta} & -\frac{a_{2}(t)^{2} \bar{x}_{2} \bar{x}_{3}}{\Delta} & \frac{a_{2}(t)^{2}\left(a_{1}(t)^{2} a_{2}(t)^{2}+\bar{x}_{2}^{2}\right)}{\Delta}
\end{array}\right)
$$

where

$$
\Delta=\bar{x}_{3}^{2} a_{2}(t)^{4}+a_{1}(t)^{2} a_{2}(t)^{2}+\bar{x}_{2}^{2} .
$$

Together with dilaton

$$
\bar{\Phi}\left(t, \bar{x}_{2}, \bar{x}_{3}\right)=\beta t-\frac{1}{2} \ln \Delta
$$

background (4.4) satisfies the Generalized Supergravity Equations (2.17)-(2.19) for $\overline{\mathcal{J}}=$ $(0,1,0,0)$. On the other hand $\operatorname{Div} \Theta=(0,2,0,0)$. 


\subsection{Bianchi $V I_{\kappa}$ cosmology}

Now we are ready to study Poisson-Lie T-pluralities of the most complicated curved cosmology invariant with respect to Bianchi $V I_{\kappa}$. Its Lie algebra is contained in semi-Abelian six-dimensional Manin triple ${ }^{8} \mathfrak{d}=\mathfrak{b}_{V I_{\kappa}} \bowtie \mathfrak{a}$ spanned by basis $\left(T_{1}, T_{2}, T_{3}, \widetilde{T}^{1}, \widetilde{T}^{2}, \widetilde{T}^{3}\right)$ with non-trivial algebraic relations

$$
\begin{aligned}
& {\left[T_{1}, T_{2}\right]=\kappa T_{2}, \quad\left[T_{1}, T_{3}\right]=T_{3}, \quad \kappa \neq-1,} \\
& {\left[T_{1}, \widetilde{T}^{2}\right]=-\kappa \widetilde{T}^{2}, \quad\left[T_{1}, \widetilde{T}^{3}\right]=-\widetilde{T}^{3}, \quad\left[T_{2}, \widetilde{T}^{2}\right]=\kappa \widetilde{T}^{1}, \quad\left[T_{3}, \widetilde{T}^{3}\right]=\widetilde{T}^{1}}
\end{aligned}
$$

Note that for $\kappa=0$, or $\kappa=1$, these are comutation relations of $\mathfrak{b}_{I I I}$, or $\mathfrak{b}_{V}$ respectively. The case $\kappa=-1$ was treated separately in section 4.1 . The group $\mathscr{B}_{V I_{\kappa}}$ is not semisimple and trace of its structure constants does not vanish. Lie algebra of the Drinfel'd double $\left(\mathscr{B}_{V I_{\kappa}} \mid \mathscr{A}\right)$ admits several other Manin triples [22].

Metric of Bianchi $V I_{\kappa}$ cosmology reads [14]

$$
\mathcal{F}\left(t, y_{1}\right)=\left(\begin{array}{cccc}
-e^{-4 \Phi(t)} a_{1}(t)^{2} a_{2}(t)^{2} a_{3}(t)^{2} & 0 & 0 & 0 \\
0 & a_{1}(t)^{2} & 0 & 0 \\
0 & 0 & e^{2 \kappa y_{1}} a_{2}(t)^{2} & 0 \\
0 & 0 & 0 & e^{2 y_{1}} a_{3}(t)^{2}
\end{array}\right)
$$

where the functions $a_{i}(t)$ have the form

$$
\begin{aligned}
& a_{1}(t)=e^{\Phi(t)}\left(\frac{p_{1}}{\kappa+1}\right)^{\frac{\kappa^{2}+1}{(\kappa+1)^{2}}} e^{\frac{(\kappa-1) p_{2} t}{2(\kappa+1)}} \sinh ^{-\frac{\kappa^{2}+1}{(\kappa+1)^{2}}}\left(p_{1} t\right), \\
& a_{2}(t)=e^{\Phi(t)}\left(\frac{p_{1}}{\kappa+1}\right)^{\frac{\kappa}{\kappa+1}} e^{\frac{p_{2} t}{2}} \sinh ^{-\frac{\kappa}{\kappa+1}}\left(p_{1} t\right), \\
& a_{3}(t)=e^{\Phi(t)}\left(\frac{p_{1}}{\kappa+1}\right)^{\frac{1}{\kappa+1}} e^{-\frac{p_{2} t}{2}} \sinh ^{-\frac{\kappa}{\kappa+1}}\left(p_{1} t\right),
\end{aligned}
$$

and dilaton is $\Phi(t)=\beta t$. The background (4.8) is invariant with respect to symmetry generated by left-invariant vector fields

$$
V_{1}=\partial_{y_{1}}-\kappa y_{2} \partial_{y_{2}}-y_{3} \partial_{y_{3}}, \quad V_{2}=\partial_{y_{2}}, \quad V_{3}=\partial_{y_{3}}
$$

satisfying (4.6). The beta function equations reduce to condition

$$
\beta^{2}=\frac{\left(\kappa^{2}+\kappa+1\right) p_{1}^{2}}{(\kappa+1)^{2}}-\frac{p_{2}^{2}}{4} .
$$

The background is torsionless and for $\beta=0$ also Ricci flat.

\footnotetext{
${ }^{8}$ Linear transformation between basis of $\mathfrak{b}_{V I_{\kappa}} \bowtie \mathfrak{a}$ used in this paper and vectors $X_{i}, \widetilde{X}_{j}$ that span Lie algebra of Drinfel'd double $\left(6_{a} \mid 1\right)$ in $[22]$ is

$$
T_{1}=-\frac{1}{1+a} X_{1}, \quad T_{2}=X_{2}-X_{3}, \quad T_{3}=X_{2}+X_{3}, \quad \kappa=\frac{a-1}{a+1}, a>0 .
$$


Formula (2.20) for new dilatons then reads

$$
\widehat{\Phi}(\hat{x}, \bar{x})=\beta t-\frac{1}{2}(1+\kappa) y_{1}(\hat{x}, \bar{x})-\frac{1}{2} \ln |\operatorname{det}[(N+\widehat{\Pi}(\hat{x}) M) \widehat{a}(\hat{x})]|
$$

so that, it is again necessary to solve $y_{1}$ from (2.22) and find how it depends on $\hat{x}$ and $\bar{x}$.

\subsubsection{Transformation of $\mathfrak{b}_{V I_{\kappa}} \bowtie \mathfrak{a}$ to $\mathfrak{b}_{V I_{\kappa}} \bowtie \mathfrak{b}_{I I}$ and to its dual}

Manin triple $\mathfrak{d}=\mathfrak{b}_{V I_{\kappa}} \bowtie \mathfrak{b}_{I I}$, which belongs to decompositions of the Lie algebra of $\left(\mathscr{B}_{V I_{\kappa}} \mid \mathscr{A}\right)$, is spanned by $\left(\widehat{T}_{1}, \widehat{T}_{2}, \widehat{T}_{3}, \bar{T}^{1}, \bar{T}^{2}, \bar{T}^{3}\right)$ with algebraic relations

$$
\begin{array}{rlrl}
{\left[\widehat{T}_{1}, \widehat{T}_{2}\right]} & =\kappa \widehat{T}_{2}, & & {\left[\widehat{T}_{1}, \widehat{T}_{3}\right]=\widehat{T}_{3},} \\
{\left[\widehat{T}_{1}, \bar{T}^{2}\right]=\widehat{T}_{3}-\kappa \bar{T}^{2},} & {\left[\widehat{T}_{1}, \bar{T}^{3}\right]=-\widehat{T}_{2}-\bar{T}^{3},} & \\
{\left[\widehat{T}_{2}, \bar{T}^{2}\right]=\kappa \bar{T}^{1},} & {\left[\widehat{T}_{3}, \bar{T}^{3}\right]=\bar{T}^{1},} & {\left[\bar{T}^{2}, \bar{T}^{3}\right]=\bar{T}^{1}}
\end{array}
$$

Left-invariant fields of $\widehat{\mathscr{G}}$ and $\overline{\mathscr{G}}$ are

$$
\begin{array}{lll}
\widehat{V}_{1}=\partial_{\hat{x}_{1}}-\kappa \hat{x}_{2} \partial_{\hat{x}_{2}}-\hat{x}_{3} \partial_{\hat{x}_{3},} & \widehat{V}_{2}=\partial_{\hat{x}_{2}}, & \widehat{V}_{3}=\partial_{\hat{x}_{3}}, \\
\bar{V}_{1}=\partial_{\bar{x}_{1}}, & \bar{V}_{2}=-\bar{x}_{3} \partial_{\bar{x}_{1}}+\partial_{\bar{x}_{2}}, & \bar{V}_{3}=\partial_{\bar{x}_{3}} .
\end{array}
$$

There are two different linear mappings (2.8) between $\mathfrak{b}_{V I_{\kappa}} \bowtie \mathfrak{a}$ and $\mathfrak{b}_{V I_{\kappa}} \bowtie \mathfrak{b}_{I I}$. One of them is given by the matrix

$$
C_{1}=\left(\begin{array}{cccccc}
-1 & c_{12} & c_{13} & c_{12} c_{15}+c_{13} c_{16} & c_{15} & c_{16} \\
0 & 0 & 0 & \frac{c_{12}}{c_{52}} & \frac{1}{c_{52}} & 0 \\
0 & 0 & 0 & (\kappa+1) c_{13} c_{56} & 0 & (\kappa+1) c_{56} \\
0 & 0 & 0 & -1 & 0 & 0 \\
0 & c_{52} & 0 & c_{15} c_{52}+c_{13} c_{56} & 0 & c_{56} \\
0 & 0 & \frac{1}{(\kappa+1) c_{56}} & \frac{c_{16}-\frac{c_{12}}{c_{52}}}{\kappa+1} & -\frac{1}{(\kappa+1) c_{52}} & 0
\end{array}\right)
$$

and the second is

$$
C_{2}=\left(\begin{array}{cccccc}
1 & c_{12} & c_{13} & -c_{12} c_{15}-c_{13} c_{16} & c_{15} & c_{16} \\
0 & \frac{1}{c_{55}} & 0 & -\frac{c_{15}}{c_{55}} & 0 & 0 \\
0 & 0 & (\kappa+1) c_{53} & -(\kappa+1) c_{16} c_{53} & 0 & 0 \\
0 & 0 & 0 & 1 & 0 & 0 \\
0 & 0 & c_{53} & -c_{16} c_{53}-c_{12} c_{55} & c_{55} & 0 \\
0-\frac{1}{(\kappa+1) c_{55}} & 0 & \frac{\frac{c_{5}}{c_{55}}-\frac{c_{13}}{c_{53}}}{\kappa+1} & 0 & \frac{1}{(\kappa+1) c_{53}}
\end{array}\right) .
$$

Tensors $\widehat{\mathcal{F}}$ on $\widehat{\mathscr{G}}=\mathscr{G}=\mathscr{B}_{V I_{\kappa}}$ calculated from these general forms are rather extensive. Nevertheless, up to change of coordinates and gauge shift the backgrounds coming from $C_{1}$ are equivalent to

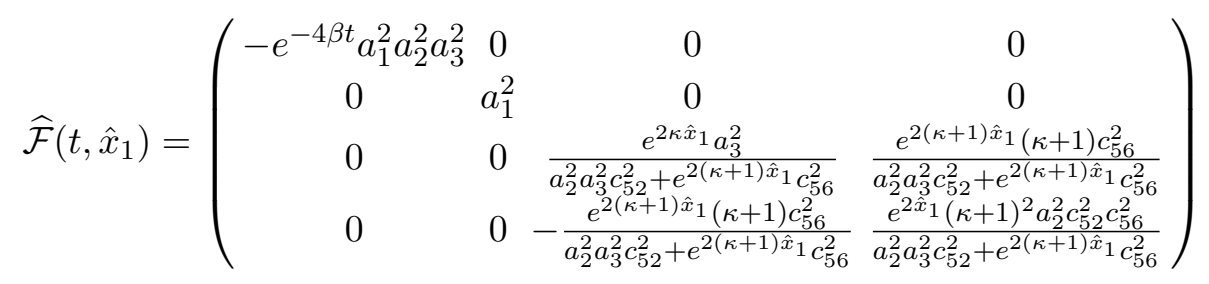


that can be obtained from $C_{1}$ when all constants $c_{i j}$ except $c_{52}$ and $c_{56}$ are set to zero. Since $y_{1}=-\hat{x}_{1}$, we get

$$
\widehat{\Phi}\left(t, \hat{x}_{1}\right)=\beta t-\frac{1}{2} \ln \left(\frac{c_{52}^{2} a_{2}(t)^{2} a_{3}(t)^{2} e^{-2 \hat{x}_{1}(\kappa+1)}+c_{56}^{2}}{(\kappa+1) c_{52} c_{56}}\right)
$$

and $\widehat{\mathcal{J}}=(0,0,0,0)=\operatorname{Div} \Theta$. Dilaton together with (4.12) satisfies beta function equations if the condition (4.10) holds. Coordinate transformation brings background on $\overline{\mathscr{G}}=\mathscr{B}_{I I}$ calculated from $D_{0} \cdot C_{1}$ to the form

$$
\overline{\mathcal{F}}\left(t, \bar{x}_{2}, \bar{x}_{3}\right)=\left(\begin{array}{cccc}
-e^{-4 \beta t} a_{1}^{2} a_{2}^{2} a_{3}^{2} & 0 & 0 & 0 \\
0 & \frac{1}{\Delta} & \frac{(\kappa+1) a_{2}^{2} \bar{x}_{2}}{\kappa c_{56} \Delta} & \frac{a_{3}^{2} \bar{x}_{3}}{(\kappa+1) c_{56} \Delta} \\
0 & -\frac{(\kappa+1) a_{2}^{2} \bar{x}_{2}}{\kappa c_{56} \Delta} & \frac{a_{2}^{2}\left((\kappa+1)^{2} a_{1}^{2} c_{56}^{2}+a_{3}^{2} \bar{x}_{3}^{2}\right)}{\kappa^{2} c_{56}^{2} \Delta} & \frac{-c_{56} \Delta-(\kappa+1) a_{2}^{2} a_{3}^{2} c_{52} \bar{x}_{2} \bar{x}_{3}}{\kappa(\kappa+1) c_{52} c_{6}^{2} \Delta} \\
0 & -\frac{a_{3}^{2} \bar{x}_{3}}{(\kappa+1) c_{56} \Delta} & \frac{c_{56} \Delta-(\kappa+1) a_{2}^{2} a_{3}^{2} c_{52} \bar{x}_{2} \bar{x}_{3}}{\kappa(\kappa+1) c_{52} c_{56}^{2} \Delta} & \frac{a_{3}^{2}\left(a_{1}^{2} c_{56}^{2} a_{2}^{2} \bar{x}_{2}^{2}\right)}{c_{56}^{2} \Delta}
\end{array}\right)
$$

where

$$
\Delta=(\kappa+1)^{2} a_{1}^{2} c_{56}^{2}+(\kappa+1)^{2} a_{2}^{2} \bar{x}_{2}^{2}+a_{3}^{2} \bar{x}_{3}^{2} .
$$

With the corresponding dilaton

$$
\bar{\Phi}\left(t, \bar{x}_{2}, \bar{x}_{3}\right)=\beta t-\frac{1}{2} \log \left(\frac{\Delta}{(\kappa+1) c_{52} c_{56}}\right)
$$

the background $\overline{\mathcal{F}}$ satisfies Generalized Supergravity Equations with $\overline{\mathcal{J}}=(0,0,0,0)$ obtained from $y_{1}=-\hat{x}_{1}$ and (2.24). However, $\operatorname{Div} \Theta$ is nontrivial and depends on $a_{2}(t) a_{3}(t)$.

Transformation of Manin triple given by $C_{2}$ gives similar results. All constants $c_{i j}$ except $c_{53}$ and $c_{55}$ can be set to zero, and the only relevant difference is that in this case $y_{1}=\hat{x}_{1}$, so the Generalized Supergravity Equations are satisfied with $\widehat{\mathcal{J}}=(0,0,0,0)$ and $\overline{\mathcal{J}}=(0, \kappa+1,0,0)$.

\subsubsection{Transformation of $\mathfrak{b}_{V I_{\kappa}} \bowtie \mathfrak{a}$ to $\mathfrak{b}_{V I_{\kappa}} \bowtie \mathfrak{b}_{V I_{-\kappa} . i i i}$ and to its dual}

The Manin triple $\mathfrak{b}_{V I_{\kappa}} \bowtie \mathfrak{b}_{V I_{-\kappa} . i i i}$ is another Manin triple of the Drinfel'd double $\left(\mathscr{B}_{V I_{\kappa}} \mid \mathscr{A}\right)$. It corresponds to $\left(6_{a} \mid 6_{1 / a} . i i i\right)$ in [22] by the transformation (4.5). It is spanned by $\left(\widehat{T}_{1}, \widehat{T}_{2}, \widehat{T}_{3}, \bar{T}^{1}, \bar{T}^{2}, \bar{T}^{3}\right)$ with algebraic relations

$$
\begin{aligned}
& {\left[\widehat{T}_{1}, \widehat{T}_{2}\right]=\kappa \widehat{T}_{2}, \quad\left[\widehat{T}_{1}, \widehat{T}_{3}\right]=\widehat{T}_{3}, \quad\left[\widehat{T}_{1}, \bar{T}^{1}\right]=\widehat{T}_{3}, \quad\left[\widehat{T}_{1}, \bar{T}^{2}\right]=-\kappa \bar{T}^{2},} \\
& {\left[\widehat{T}_{1}, \bar{T}^{3}\right]=-\widehat{T}_{1}-\bar{T}^{3}, \quad\left[\widehat{T}_{2}, \bar{T}^{2}\right]=\kappa\left(-\widehat{T}_{3}+\bar{T}^{1}\right), \quad\left[\widehat{T}_{2}, \bar{T}^{3}\right]=\kappa \widehat{T}_{2},} \\
& {\left[\widehat{T}_{3}, \bar{T}^{3}\right]=\bar{T}^{1}, \quad\left[\bar{T}^{1}, \bar{T}^{3}\right]=\bar{T}^{1}, \quad\left[\bar{T}^{2}, \bar{T}^{3}\right]=-\kappa \bar{T}^{2} .}
\end{aligned}
$$

Left-invariant fields of $\widehat{\mathscr{G}}$ and $\overline{\mathscr{G}}$ are

$$
\begin{array}{rlrl}
\widehat{V}_{1} & =\partial_{\hat{x}_{1}}-\kappa \hat{x}_{2} \partial_{\hat{x}_{2}}-\hat{x}_{3} \partial_{\hat{x}_{3}}, & \widehat{V}_{2}=\partial_{\hat{x}_{2}}, & \widehat{V}_{3}=\partial_{\hat{x}_{3}}, \\
\bar{V}_{1}=e^{-\bar{x}_{3}} \partial_{\bar{x}_{1}}, & \bar{V}_{2}=e^{\kappa \bar{x}_{3}} \partial_{\bar{x}_{2}}, & \bar{V}_{3}=\partial_{\bar{x}_{3}} .
\end{array}
$$


There are two different Poisson-Lie T-pluralities between $\mathfrak{b}_{V I_{\kappa}} \bowtie \mathfrak{a}$ and $\mathfrak{b}_{V I_{\kappa}} \bowtie$ $\mathfrak{b}_{V I_{-\kappa} . i i i}$. One of them is given by the matrix

$$
C_{1}=\left(\begin{array}{cccccc}
-1 & c_{12} & c_{13} & c_{12} c_{15}+c_{13} c_{16} & c_{15} & c_{16} \\
0 & 0 & 0 & \frac{c_{12}}{c_{52}} & \frac{1}{c_{52}} & 0 \\
0 & 0 & 0 & c_{13} c_{36} & 0 & c_{36} \\
0 & 0 & 0 & c_{13} c_{36}-1 & 0 & c_{36} \\
0 & c_{52} & 0 & c_{15} c_{52} & 0 & 0 \\
1 & -c_{12} & \frac{1}{c_{36}}-c_{13} & c_{16}\left(\frac{1}{c_{36}}-c_{13}\right)-c_{12} c_{15} & -c_{15} & -c_{16}
\end{array}\right)
$$

and the other one is

$$
C_{2}=\left(\begin{array}{cccccc}
1 & c_{12} & c_{13} & -c_{12} c_{15}-c_{13} c_{16} & c_{15} & c_{16} \\
0 & c_{22} & 0 & -c_{15} c_{22} & 0 & 0 \\
0 & 0 & c_{33} & -c_{16} c_{33} & 0 & 0 \\
0 & 0 & c_{33} & 1-c_{16} c_{33} & 0 & 0 \\
0 & 0 & 0 & -\frac{c_{12}}{c_{22}} & \frac{1}{c_{22}} & 0 \\
-1 & -c_{12} & -c_{13} & c_{12} c_{15}+c_{13}\left(c_{16}-\frac{1}{c_{33}}\right) & -c_{15} & \frac{1}{c_{33}}-c_{16}
\end{array}\right) .
$$

General forms of tensors and dilatons $\widehat{\mathcal{F}}, \widehat{\Phi}$ on $\widehat{\mathscr{G}}=\mathscr{G}=\mathscr{B}_{V I_{\kappa}}$ and $\overline{\mathcal{F}}, \bar{\Phi}$ on $\overline{\mathscr{G}}=$ $\mathscr{B}_{V I_{-\kappa} . i i i}$ are too extensive to display. Setting $c_{12}=c_{13}=c_{15}=c_{16}=0$ and $c_{52}=c_{36}=1$ in $C_{1}$ we get

$$
\widehat{\mathcal{F}}\left(t, \hat{x}_{1}, \hat{x}_{2}\right)=\left(\begin{array}{cccc}
-e^{-4 \beta t} a_{1}^{2} a_{2}^{2} a_{3}^{2} & 0 & 0 & 0 \\
0 & \frac{a_{1}^{2}\left(a_{2}^{2} a_{3}^{2}+e^{2(\kappa+1) \hat{x}_{1}} \kappa^{2} \hat{x}_{2}^{2}\right)}{\Delta} & \frac{e^{2(\kappa+1) \hat{x}} \hat{x}_{1} \kappa a_{1}^{2} \hat{x}_{2}}{\Delta} & \frac{e^{2 \hat{x}_{1}} a_{1}^{2} a_{2}^{2}}{\Delta} \\
0 & \frac{e^{2(\kappa+1) \hat{x}_{1}} \kappa a_{1}^{2} \hat{x}_{2}}{\Delta} & \frac{e^{2 \kappa \hat{x}_{1}\left(e^{2 \hat{x}} 1 a_{1}^{2}+a_{3}^{2}\right)}}{\Delta} & -\frac{e^{2(\kappa+1) \hat{x}_{1}} \kappa \hat{x}_{2}}{\Delta} \\
0 & -\frac{e^{2 \hat{x}_{1}} a_{1}^{2} a_{2}^{2}}{\Delta} & \frac{e^{2(\kappa+1) \hat{x}_{1} \hat{x}_{2}}}{\Delta} & \frac{e^{2 x_{1}} a_{2}^{2}}{\Delta}
\end{array}\right)
$$

where

$$
\Delta=e^{2 \hat{x}_{1}} a_{1}^{2} a_{2}^{2}+a_{3}^{2} a_{2}^{2}+e^{2(\kappa+1) \hat{x}_{1}} \kappa^{2} \hat{x}_{2}^{2}
$$

and

$$
\widehat{\Phi}\left(t, \hat{x}_{1}, \hat{x}_{2}\right)=\beta t-\frac{1}{2} \log \left(-e^{-2(\kappa+1) \hat{x}_{1}} \Delta\right) .
$$

Generalized Supergravity Equations with $\widehat{\mathcal{J}}=(0,0,0, \kappa)$ obtained from $(2.23)$ and $y_{1}=$ $-\hat{x}_{1}+\bar{x}_{3}$ are satisfied under the condition (4.10).

Dual transformation gives

$\overline{\mathcal{F}}\left(t, \bar{x}_{1}, \bar{x}_{2}, \bar{x}_{3}\right)=\left(\begin{array}{cccc}\frac{-e^{4 t \beta}}{a_{1}^{2} a_{2}^{2} a_{3}^{2}} & 0 & 0 & 0 \\ 0 & \frac{a_{1}^{2} a_{3}^{2}+\bar{x}_{1}^{2}}{a_{1}^{2}+a_{3}^{2}} & -\frac{\kappa\left(a_{3}^{2}+\bar{x}_{1}\right) \bar{x}_{2}}{a_{1}^{2}+a_{3}^{2}} & \frac{e^{-\bar{x}_{3}} a_{1}^{2}+\left(e^{-\bar{x}_{3}}-1\right) a_{3}^{2}-\bar{x}_{1}}{a_{1}^{2}+a_{3}^{2}} \\ 0 & \frac{\kappa\left(a_{3}^{2}-\bar{x}_{1}\right) \bar{x}_{2}}{a_{1}^{2}+a_{3}^{2}} & \frac{a_{1}^{2}+a_{3}^{2}+\kappa^{2} a_{2}^{2} \bar{x}_{2}^{2}}{a_{2}^{2}\left(a_{1}^{2}+a_{3}^{2}\right)} & \frac{\kappa \bar{x}_{2}}{a_{1}^{2}+a_{3}^{2}} \\ 0 & -\frac{e^{-\bar{x}_{3}} a_{1}^{2}+\left(-1+e^{-\bar{x}_{3}}\right) a_{3}^{2}+\bar{x}_{1}}{a_{1}^{2}+a_{3}^{2}} & \frac{\kappa \bar{x}_{2}}{a_{1}^{2}+a_{3}^{2}} & \frac{1}{a_{1}^{2}+a_{3}^{2}}\end{array}\right)^{-1}$

and

$$
\bar{\Phi}\left(t, \bar{x}_{2}, \bar{x}_{3}\right)=\beta t-\frac{1}{2} \log \left(a_{1}^{2}+\kappa^{2} \bar{x}_{2}^{2} a_{2}^{2}+\left(e^{\bar{x}_{3}}-1\right)^{2} a_{3}^{2}\right) .
$$


Generalized Supergravity Equations with $\overline{\mathcal{J}}=(0,0,0,0)$ are satisfied under the condition (4.10). Vectors $\widehat{\mathcal{J}}, \overline{\mathcal{J}}$ do not agree with $\operatorname{Div} \Theta$.

Transformation of Manin triple given by particular form of $C_{2}$ where

$$
c_{12}=c_{13}=c_{15}=c_{16}=0, c_{22}=c_{33}=1
$$

gives again rather extensive tensors and dilatons. The only relevant difference is that now $y_{1}=\hat{x}_{1}-\bar{x}_{3}$ and the Generalized Supergravity Equations are satisfied with $\widehat{\mathcal{J}}=(0,0,0,-1)$ and $\overline{\mathcal{J}}=\left(0,(\kappa+1) e^{-\bar{x}_{3}}, 0,0\right)$. Vector $\overline{\mathcal{J}}$ does not agree with Div $\Theta$ that depends on the product $a_{1}(t) a_{3}(t)$.

\section{Conclusions}

We have presented many examples of Poisson-Lie T-plurality transformation acting on flat or curved backgrounds invariant with respect to Bianchi groups $\mathscr{B}_{I I I}, \mathscr{B}_{V}, \mathscr{B}_{V I_{\kappa}}$ and $\mathscr{B}_{V I_{-1}}$. Coresponding dilatons were found using formulas (2.20), (2.21). In many cases the dilatons were non-local in the sense that they were both functions of coordinates $\hat{x}$ on $\widehat{\mathscr{G}}$ and $\bar{x}$ on $\overline{\mathscr{G}}$. We have shown how to deal with this issue if the dependency is linear. This partially resolves the puzzle explained in 2.3.

It turns out that plural backgrounds and dilatons often do not satisfy the usual beta function equations but Generalized Supergravity Equations provided the supplementary vector field $\mathcal{J}$ is computed using formulas (2.23) or (2.24) presented in section 2.3. The formulas were repeatedly checked not only for the examples presented here, but also for other Manin triples given in [22] and their embedings into $\mathfrak{d}$. All tested backgrounds and dilatons obtained by Poisson-Lie transformations (2.13), (2.14), (2.20), (2.21) satisfy Generalized Supergravity Equations.

As we noted in the Introduction, complete classification of plural models is beyond the scope of the paper as there are too many cases to discuss. Therefore, we present Manin triples that demonstrate important properties of plural sigma models, dilatons and vectors $\mathcal{J}$. The examples show that vector fields $\mathcal{J}$ need not be constant as it turns out in cases of plurality $\mathfrak{b}_{I I I} \bowtie \mathfrak{a}$ to $\mathfrak{b}_{\text {IIIiii }} \bowtie \mathfrak{b}_{I I I}, \mathfrak{b}_{V} \bowtie \mathfrak{a}$ to $\mathfrak{b}_{V i i} \bowtie \mathfrak{b}_{V I_{-1}}$ and $\mathfrak{b}_{V I_{\kappa}} \bowtie \mathfrak{a}$ to $b_{V I_{\kappa}} \bowtie \mathfrak{b}_{V I_{-\kappa} . i i i}$. Let us note that vector fields $\mathcal{J}$ are Killing vectors of corresponding backgrounds in spite of the fact that they are linear combinations of left-invariant fields of corresponding groups that satisfy condition (2.25) with generally nonvanishing right-hand side. Beside that, we have shown that the alternative formula (2.26) for the supplementary vector $\mathcal{J}$ developed for the (non-)Abelian T-duality does not work in general for Poisson-Lie T-plurality.

Open Access. This article is distributed under the terms of the Creative Commons Attribution License (CC-BY 4.0), which permits any use, distribution and reproduction in any medium, provided the original author(s) and source are credited. 


\section{References}

[1] T.H. Buscher, A symmetry of the string background field equations, Phys. Lett. B 194 (1987) 59 [INSPIRE].

[2] X.C. de la Ossa and F. Quevedo, Duality symmetries from non-Abelian isometries in string theory, Nucl. Phys. B 403 (1993) 377 [hep-th/9210021] [INSPIRE].

[3] K. Sfetsos and D.C. Thompson, On non-Abelian T-dual geometries with Ramond fluxes, Nucl. Phys. B 846 (2011) 21 [arXiv: 1012.1320] [InSPIRE].

[4] Y. Lozano, E. Ó Colgáin, K. Sfetsos and D.C. Thompson, Non-Abelian T-duality, Ramond fields and coset geometries, JHEP 06 (2011) 106 [arXiv:1104.5196] [INSPIRE].

[5] G. Itsios, Y. Lozano, J. Montero and C. Núñez, The AdS $S_{5}$ non-Abelian T-dual of Klebanov-Witten as a $N=1$ linear quiver from M5-branes, JHEP 09 (2017) 038 [arXiv: 1705.09661] [INSPIRE].

[6] G. Itsios, H. Nastase, C. Núñez, K. Sfetsos and S. Zacarías, Penrose limits of Abelian and non-Abelian T-duals of $A d S_{5} \times S^{5}$ and their field theory duals, JHEP 01 (2018) 071 [arXiv: 1711.09911] [INSPIRE].

[7] M. Gasperini, R. Ricci and G. Veneziano, A problem with non-Abelian duality?, Phys. Lett. B 319 (1993) 438 [hep-th/9308112] [INSPIRE].

[8] E. Álvarez, L. Álvarez-Gaumé and Y. Lozano, On non-Abelian duality, Nucl. Phys. B 424 (1994) 155 [hep-th/9403155] [INSPIRE].

[9] R. Borsato and L. Wulff, Integrable deformations of T-dual $\sigma$ models, Phys. Rev. Lett. 117 (2016) 251602 [arXiv: 1609.09834] [INSPIRE].

[10] S. Demulder, F. Hassler and D.C. Thompson, Doubled aspects of generalised dualities and integrable deformations, JHEP 02 (2019) 189 [arXiv:1810.11446] [INSPIRE].

[11] G. Arutyunov, S. Frolov, B. Hoare, R. Roiban and A.A. Tseytlin, Scale invariance of the $\eta$-deformed $A d S_{5} \times S^{5}$ superstring, T-duality and modified type-II equations, Nucl. Phys. B 903 (2016) 262 [arXiv: 1511.05795] [INSPIRE].

[12] L. Wulff and A.A. Tseytlin, $\kappa$-symmetry of superstring $\sigma$-model and generalized $10 d$ supergravity equations, JHEP 06 (2016) 174 [arXiv: 1605.04884] [INSPIRE].

[13] B. Hoare and A.A. Tseytlin, Homogeneous Yang-Baxter deformations as non-Abelian duals of the $A d S_{5}$ б-model, J. Phys. A 49 (2016) 494001 [arXiv:1609.02550] [InSPIRE].

[14] M. Hong, Y. Kim and E. Ó Colgáin, On non-Abelian T-duality for non-semisimple groups, Eur. Phys. J. C 78 (2018) 1025 [arXiv:1801.09567] [InSPIRE].

[15] N.A. Batakis and A.A. Kehagias, Anisotropic space-times in homogeneous string cosmology, Nucl. Phys. B 449 (1995) 248 [hep-th/9502007] [InSPIRE].

[16] L. Hlavatý and I. Petr, Poisson-Lie identities and dualities of Bianchi cosmologies, Eur. Phys. J. C 79 (2019) 855 [arXiv:1905.13627] [InSPIRE].

[17] D. Lüst and D. Osten, Generalised fluxes, Yang-Baxter deformations and the $O(d, d)$ structure of non-Abelian T-duality, JHEP 05 (2018) 165 [arXiv:1803.03971] [INSPIRE].

[18] C. Klimčík and P. Ševera, Dual non-Abelian duality and the Drinfeld double, Phys. Lett. B 351 (1995) 455 [hep-th/9502122] [INSPIRE].

[19] R. Von Unge, Poisson Lie T plurality, JHEP 07 (2002) 014 [hep-th/0205245] [INSPIRE]. 
[20] C. Klimčík, Poisson-Lie T duality, Nucl. Phys. Proc. Suppl. 46 (1996) 116 [hep-th/9509095] [INSPIRE].

[21] Y. Sakatani, Type II DFT solutions from Poisson-Lie T-duality/plurality, arXiv:1903.12175 [INSPIRE].

[22] L. Šnobl and L. Hlavatý, Classification of six-dimensional real Drinfel'd doubles, Int. J. Mod. Phys. A 17 (2002) 4043 [math.QA/0202209] [INSPIRE].

[23] L. Hlavatý and L. Šnobl, Poisson-Lie T-plurality of three-dimensional conformally invariant $\sigma$-models. II. Nondiagonal metrics and dilaton puzzle, JHEP 10 (2004) 045 [hep-th/0408126] [INSPIRE].

[24] T. Araujo, I. Bakhmatov, E. Ó Colgáin, J. Sakamoto, M.M. Sheikh-Jabbari and K. Yoshida, Yang-Baxter $\sigma$-models, conformal twists and noncommutative Yang-Mills theory, Phys. Rev. D 95 (2017) 105006 [arXiv: 1702.02861] [INSPIRE].

[25] T. Araujo, I. Bakhmatov, E. Ó Colgáin, J.-I. Sakamoto, M.M. Sheikh-Jabbari and K. Yoshida, Conformal twists, Yang-Baxter $\sigma$-models \& holographic noncommutativity, J. Phys. A 51 (2018) 235401 [arXiv:1705.02063] [inSPIRE].

[26] T. Araujo, E. Ó Colgáin, J. Sakamoto, M.M. Sheikh-Jabbari and K. Yoshida, I in generalized supergravity, Eur. Phys. J. C 77 (2017) 739 [arXiv:1708.03163] [INSPIRE].

[27] L. Hlavatý, I. Petr and V. Štěpán, Poisson-Lie T-plurality with spectators, J. Math. Phys. 50 (2009) 043504.

[28] L. Hlavatý and F. Petrásek, On uniqueness of T-duality with spectators, Int. J. Mod. Phys. A 31 (2016) 1650143 [arXiv: 1606.02522] [INSPIRE].

[29] S. Majid, Foundations of quantum group theory, Cambridge University Press, Cambridge, U.K. (1995).

[30] C.G. Callan Jr., E.J. Martinec, M.J. Perry and D. Friedan, Strings in background fields, Nucl. Phys. B 262 (1985) 593 [INSPIRE].

[31] W. Mück, Generalized supergravity equations and generalized Fradkin-Tseytlin counterterm, JHEP 05 (2019) 063 [arXiv: 1904.06126] [INSPIRE].

[32] J.-I. Sakamoto, Y. Sakatani and K. Yoshida, Homogeneous Yang-Baxter deformations as generalized diffeomorphisms, J. Phys. A 50 (2017) 415401 [arXiv:1705.07116] [INSPIRE].

[33] M. Gasperini and R. Ricci, Homogeneous conformal string backgrounds, Class. Quant. Grav. 12 (1995) 677 [hep-th/9501055] [INSPIRE].

[34] S. Elitzur, A. Giveon, E. Rabinovici, A. Schwimmer and G. Veneziano, Remarks on non-Abelian duality, Nucl. Phys. B 435 (1995) 147 [hep-th/9409011] [INSPIRE].

[35] L. Hlavatý and I. Petr, Plane-parallel waves as duals of the flat background, Class. Quant. Grav. 32 (2015) 035005 [arXiv: 1406.0971] [InSPIRE].

[36] F. Petrásek, L. Hlavatý and I. Petr, Plane-parallel waves as duals of the flat background II: T-duality with spectators, Class. Quant. Grav. 34 (2017) 155003 [arXiv:1612.08015] [INSPIRE].

[37] L. Hlavatý, I. Petr and F. Petrásek, Plane-parallel waves as duals of the flat background III: T-duality with torsionless B-field, Class. Quant. Grav. 35 (2018) 075012 [arXiv: 1711.08688] [INSPIRE]. 